Geometry $\&$ Topology Monographs

Volume 1: The Epstein birthday schrift

Pages 511-549

\title{
Shapes of polyhedra and triangulations of the sphere
}

\author{
WiLliam P THURSTON
}

\begin{abstract}
The space of shapes of a polyhedron with given total angles less than $2 \pi$ at each of its $n$ vertices has a Kähler metric, locally isometric to complex hyperbolic space $\mathbb{C} \mathbb{H}^{n-3}$. The metric is not complete: collisions between vertices take place a finite distance from a nonsingular point. The metric completion is a complex hyperbolic cone-manifold. In some interesting special cases, the metric completion is an orbifold. The concrete description of these spaces of shapes gives information about the combinatorial classification of triangulations of the sphere with no more than 6 triangles at a vertex.
\end{abstract}

AMS Classification 51M20; 51F15, 20H15, 57M50

Keywords Polyhedra, triangulations, configuration spaces, braid groups, complex hyperbolic orbifolds

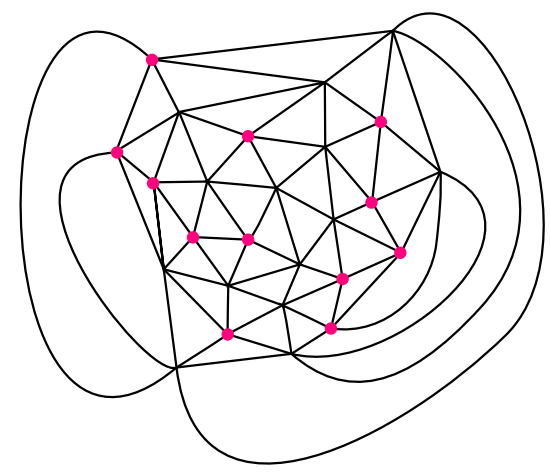

Figure 1: The twelve marked vertices of this triangulation of $S^{2}$ have five triangles while all other vertices have six. Theorem 0.1 implies that the possible triangulations satisfying this condition are parametrized, up to isomorphism, by 20-tuples of integers up to the action of a group of integer linear transformations.

\section{Introduction}

There are only three completely symmetric triangulations of the sphere: the tetrahedron, the octahedron and the icosahedron. However, finer triangulations with good geometric properties are often encountered or desired for mathematical, scientific or technological reasons, for example, the kinds of triangulations 
popularized in modern times by Buckminster Fuller and used for geodesic domes and chemical 'Buckyballs'.

There are procedures to refine and modify any triangulation of a surface until every vertex has either 5,6 or 7 triangles around it, or with more effort, so that there are only 5 or 6 triangles if the surface has positive Euler characteristic, only 6 triangles if the surface has zero Euler characteristic, or only 6 or 7 triangles if the surface has negative Euler characteristic. These conditions on triangulations are combinatorial analogues of metrics of positive, zero or negative curvature. How systematically can they be understood?

In this paper, we will develop a global theory to describe all triangulations of the $S^{2}$ such that each vertex has 6 or fewer triangles at any vertex. Here is one description:

Theorem 0.1 (Polyhedra are lattice points) There is a lattice $L$ in complex Lorentz space $C^{(1,9)}$ and a group $\Gamma$ of automorphisms, such that triangulations of non-negative combinatorial curvature are elements of $L_{+} / \Gamma$, where $L_{+}$is the set of lattice points of positive square-norm. The projective action of $\Gamma$ on complex hyperbolic space $\mathbb{C H}^{9}$ (the unit ball in $\mathbb{C}^{9} \subset \mathbb{C P}^{9}$ ) has quotient of finite volume. The square of the norm of a lattice point is the number of triangles in the triangulation.

A triangulation is non-negatively curved if there are never more than six triangles at a vertex. The theorem can be interpreted as describing certain concrete cut-and-glue constructions for creating triangulations of non-negative curvature, starting from simple and easily-classified examples. The constructions are parametrized by choices of integers, subject to certain geometric constraints. The fact that $\Gamma$ is a discrete group means that it is possible to dispense with most of the constraints, except for an algebraic condition that a certain quadratic form is positive: any choice of integer parameters can be transformed by $\Gamma$ to satisfy the geometric conditions, and the resulting triangulation is unique. Thus, the collection of all triangulations can be described either as a quotient space, in which identifications of the parameters are made algebraically, or as a fundamental domain (see section 7 ).

We will study combinatorial types of triangulations by using a metric where each triangle is a Euclidean equilateral triangle with sides of unit length. This metric is locally Euclidean everywhere except near vertices that have fewer than 6 triangles.

It is helpful to consider these metrics as a special case of metrics on the sphere which are locally Euclidean except at a finite number of points, which have 
neighborhoods locally modelled on cones. A cone of cone-angle $\theta$ is a metric space that can be formed, if $\theta \leq 2 \pi$, from a sector of the Euclidean plane between two rays that make an angle $\theta$, by gluing the two rays together. More generally, a cone of angle $\theta$ can be formed by taking the universal cover of the plane minus 0 , reinserting 0 , and then identifying modulo a transformation that "rotates" by angle $\theta$. The apex curvature of a cone of cone-angle $\theta$ is $2 \pi-\theta$.

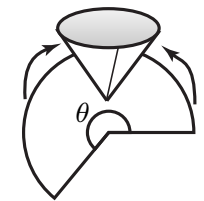

Cone angle $\theta$

A Euclidean cone metric on a surface satisfies the GaussBonnet theorem, that is, the sum of the apex curvatures is $2 \pi$ times the Euler characteristic. This fact can be derived from basic Euclidean geometry by subdividing the surface into triangles and looking at the sum of angles of all triangles grouped in two different ways, by triangle or by vertex. It can also be derived from the usual smooth Gauss-Bonnet formula by rounding off the points, replacing a tiny neighborhood of each cone point by a smooth surface (for example part of a small sphere).

Theorem 0.2 (Cone metrics form cone manifold) Let $k_{1}, k_{2}, \ldots, k_{n} \quad[n>3]$ be a collection of real numbers in the interval $(0,2 \pi)$ whose sum is $4 \pi$. Then the set of Euclidean cone metrics on the sphere with cone points of curvature $k_{i}$ and of total area 1 forms a complex hyperbolic manifold, whose metric completion is a complex hyperbolic cone manifold of finite volume. This cone manifold is an orbifold (that is, the quotient space of a discrete group) if and only if for any pair $k_{i}, k_{j}$ whose sum $s=k_{i}+k_{j}$ that is less than $2 \pi$, either

(i) $(2 \pi-s)$ divides $2 \pi$, or

(ii) $k_{i}=k_{j}$, and $(2 \pi-s) / 2=\left(\pi-k_{i}\right)$ divides $2 \pi$.

The definition of "cone-manifold" in dimensions bigger than 2 will be given later.

This turns out to be closely related to work of Picard ([6], [7]) and Mostow and Deligne ([2], [3], [5]). Picard discovered many of the orbifolds; his student LeVavasseur enumerated the class of groups Picard discovered, and they were further analyzed by Deligne and Mostow. Mostow discovered that condition (i) is not always required to obtain an orbifold and that (ii) is sufficient when $k_{i}=k_{j}$. However, the geometric interpretations were not apparent in these papers. It is possible to understand the quotient cone-manifolds quite concretely in terms of shapes of polyhedra.

A version of this paper has circulated for a number of years as a preprint, which for a time was circulated as a Geometry Center preprint, and later revised as 
part of the xxx mathematics archive. In view of this history, some time warp is inevitable: for some parts of this paper, others may have have done further work that is not here taken into account. I would like to thank Derek Holt, Igor Rivin, Chih-Han Sah and Rich Schwarz for mathematical comments and corrections that I hope I have taken into account.

\section{Triangulations of a hexagon}

Let $E$ be the standard equilateral triangulation of $\mathbb{C}$ by triangles of unit side length, where 0 and 1 are both vertices. The set Eis of vertices of $E$ are complex numbers of the form $m+p \omega$, where $\omega=1 / 2+\sqrt{-3} / 2$ is a primitive 6 th root of unity. These lattice points form a subring of $\mathbb{C}$, called the Eisenstein integers, the ring of algebraic integers in the quadratic imaginary field $\mathbb{Q}(\sqrt{-3})$.

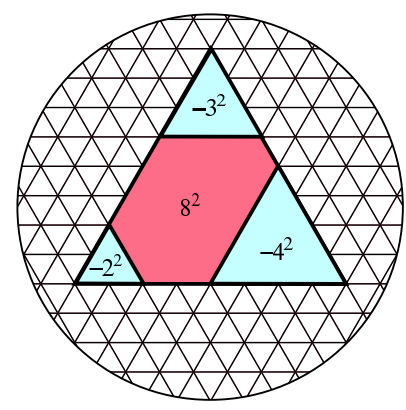

Figure 2: An Eisenstein lattice hexagon has the form of a large equilateral triangle of sidelength $n$, minus three equilateral triangles that fit inside it of sidelengths $p_{1}, p_{2}$ and $p_{3}$. An equilateral triangle of sidelength $n$ contains $n^{2}$ unit equilateral triangles, so the hexagon has $n^{2}-p_{1}^{2}-p_{2}^{2}-p_{3}^{2}$ triangles.

To warm up, we'll analyze all possible shapes of Eisenstein lattice hexagons, with vertices in Eis and sides parallel (in order) with the sides of a standard hexagon. Note that any such hexagon with $m$ triangles determines a nonnegatively curved triangulation of the sphere with $2 m$ triangles, formed by making a hexagonal envelope from two copies of the hexagon glued along the boundary.

If we circumscribe a lattice triangle $T$ about our lattice hexagon $H$, this gives a description

$$
H=T \backslash\left(S_{1} \cup S_{2} \cup S_{3}\right),
$$

where the $S_{i}$ are smaller equilateral triangles. If $T$ has sidelength $n$ and $S_{i}$ has sidelength $p_{i}$, then $H$ contains

$$
m=n^{2}-p_{1}^{2}-p_{2}^{2}-p_{3}^{2}
$$

triangles. 
All such hexagons are described by four integer parameters, subject to the 6 inequalities

$$
\begin{gathered}
p_{1} \geq 0 \quad p_{2} \geq 0 \quad p_{3} \geq 0 \\
p_{1}+p_{2} \leq n \quad p_{2}+p_{3} \leq n \quad p_{3}+p_{1} \leq n
\end{gathered}
$$

where strict inequalities give non-degenerate hexagons; if one or more inequality becomes an equality then one or more sides of the hexagon shrinks to length 0 and the 'hexagon' becomes a pentagon, quadrilateral or triangle.

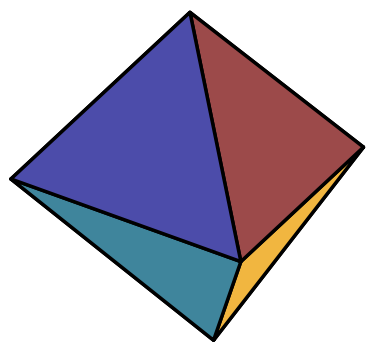

Figure 3: The space of shapes of hexagons is described by this polyhedron in hyperbolic 3-space; the faces represent hexagons degenerated to pentagons, and the edges represent degeneration to quadrilaterals. All dihedral angles are $\pi / 2$. The three mid-level vertices are ideal vertices at infinity, and represent the three ways that hexagons can become arbitrarily long and skinny, while the top and bottom are finite vertices, representing the two ways that hexagons can degenerate to equilateral triangles. The polyhedron has hyperbolic volume $.91596559417 \ldots$. .

The solutions are elements of the integer lattice inside a convex cone $C \subset$ $\mathbb{R}^{4}$. This description can be extended to non-integer parameters, which then determine a size and shape for the hexagon, but not a triangulation. Equation (1) expresses the area, measured in triangles, as a quadratic form of signature $(1,3)$. The isometry group of any such a form is $C_{2} \times \operatorname{Isom}\left(\mathbb{H}^{3}\right)$ (where $C_{2}$ denotes the cyclic group of order 2).

The possible shapes of lattice hexagons (where rescaling is allowed) are parametrized by a convex polyhedron $H \subset \mathbb{H}^{3}$ which is the projective image of the convex cone $C \subset \mathbb{R}^{(1,3)}$. This polyhedron has three ideal vertices at infinity, which represent the three directions in which shapes of hexagons can tend toward infinity, by becoming long and skinny along one of three axes. In addition, there are two finite vertices (top and bottom), representing the two ways that a hexagon can degenerate to an equilateral triangle. All dihedral angles of this hyperbolic polyhedron are $\pi / 2$. Four edges meet at each ideal vertex, while three edges meet at the finite vertices. Triangulations with $m$ triangles are represented by a discrete set $H_{m} \subset H$. Figure 4 plots the count of how many of these lattice hexagons there are with each possible area up to 1,000. One indication of the relevance of hyperbolic geometry is that the average number 

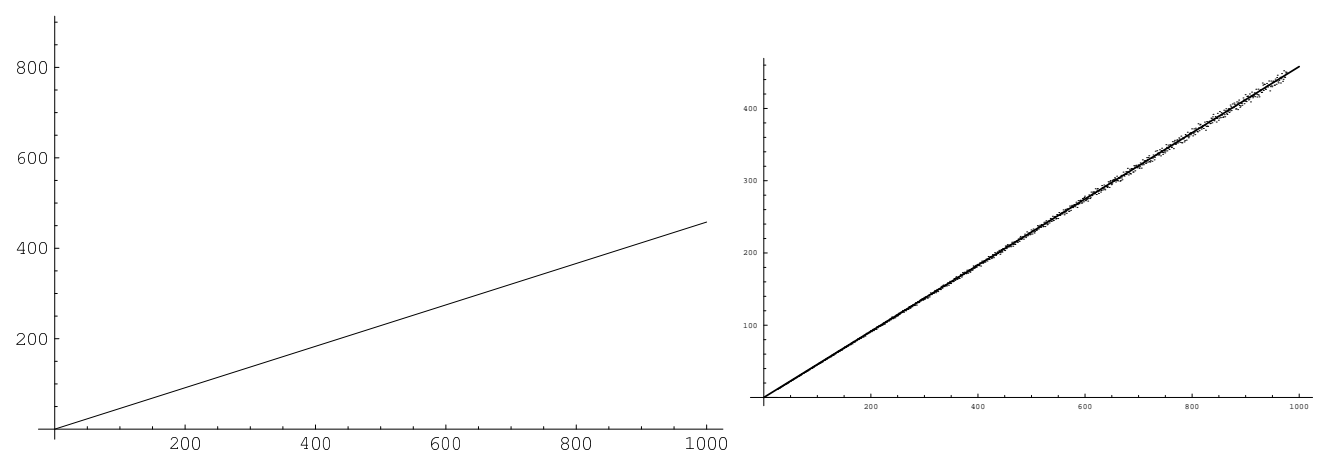

Figure 4: Left the weighted count of Eisenstein lattice hexagons containing up to 1000 triangles, using orbifold weights $1 / 2^{k}$ where $k$ is the number of sides of a hexagon of length 0 . The parameter space of shapes (figure 3) has hyperbolic volume $.91596559417 \ldots$ (1/4 that of the Whitehead Link complement), so the number of hexagons containing $m$ triangles should grow on the average as the volume of the intersection of $C / 2$ with the shell in $\mathbb{E}^{(1,3)}$ between radius $\sqrt{m}$ and $\sqrt{m+1}$, $.45798279709 \cdots * m$, as indicated. Right The same data averaged over windows of size 49.

of hexagons of a given area is well estimated by the hyperbolic volume of the parameter space.
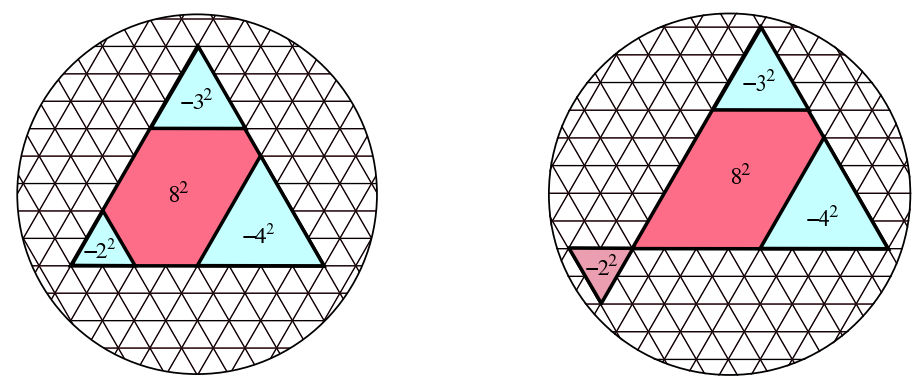

Figure 5: A butterfly operation moves one edge of a hexagon (left) across a butterflyshaped quadrilateral of 0 area, yielding a new hexagon (right) of the same area. The set of butterfly moves generate a discrete group of isometries of $\mathbb{H}^{3}$, generated by reflections in the faces of the polyhedron $H$.

It's interesting to note that $H$ is the fundamental polyhedron for a discrete group of isometries of $\mathbb{H}^{3}$, since all dihedral angles equal $\pi / 2$. This group can be interpreted in terms of not necessarily simple hexagons in the Eisenstein lattice whose sides are parallel, in order, to those of the standard hexagon. A non-simple lattice hexagon wraps with integer degree around each triangle in the plane; its total area, using these integer weights, is given by the same 
quadratic form $n^{2}-\sum p_{i}^{2}$.

Reflection in a face of the polyhedron corresponds to a 'butterfly move', which is described numerically by reversing the sign of the length of one of the edges of the hexagon, and adjusting the two neighboring lengths so that the result is a closed curve. Geometrically, the hexagon moves across a quadrilateral reminiscent of a butterfly, resulting in a new hexagon that algebraically encloses the same area as the original. Note that this operation fixes any hexagon where the given side has degenerated to have length 0 - this is one of the faces of the polyhedron $H$. The operations for two sides of the hexagon that do not meet commute with each other, and fix any shapes of hexagons where both these sides have length 0 . These shapes describe an edge of $H$, and since the reflections in adjacent faces commute, the angle must be $\pi / 2$. Two adjacent sides of the hexagon cannot both have 0 length at once, so the 9 non-adjacent pairs of sides of the hexagon correspond 1-1 to the 9 edges of $H$.

Any solution to the equation $0<m=n^{2}-p_{1}^{2}-p_{2}^{2}-p_{3}^{2}$ determines a not necessarily simple hexagon of area $m$, which projects to a point in $\mathbb{H}^{3}$. By a sequence of butterfly moves, this point can be transformed to be inside the fundamental domain $H$. The resulting point inside $H$ is uniquely determined by the initial solution and does not depend on what sequence of butterfly moves were used to get it there, since $H$ is the quotient space (quotient orbifold) for the group action as well as being its fundamental domain.

\section{Triangulations of the sphere}

Let $P\left(n ; k_{1}, k_{2}, \ldots, k_{s}\right)$ denote the set of isomorphism classes of "triangulations" of the sphere having exactly $2 n$ triangles, where for each $i$ there is one vertex incident to $6-k_{i}$ triangles, and all remaining vertices are incident to 6 triangles. This paper will be limited to the non-negatively curved cases that $0<k_{i} \leq 5$. For there to be any actual triangulations we must have $\sum_{i} k_{i}=12$. We will use the term "triangulation" throughout to refer to a space obtained by gluing together triangles by a pairing of their edges; thus, in the case $k_{i}=5$, two edges of a triangle are folded together to form a vertex incident to a single triangle. Every triangulation of the sphere has an even number of triangles.

If $T \in P\left(n ; k_{1}, \ldots, k_{s}\right)$, then there is a developing map $D_{T}$ from the universal

cover $\tilde{T}$ of $T$ minus its singular vertices into $E$. Choose any triangle of $\tilde{T}$, and map it to the triangle $\Delta(0,1, \omega)$. The developing map $D_{T}$ is now determined by a form of analytic continuation, so that it is a local isometry, mapping triangles to triangles. 


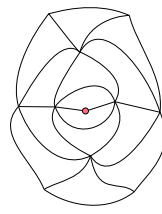

Figure 6: A vertex with 2 triangles (left) comes by folding up a $120^{\circ}$ angle (right). Equivalently, it has a neighborhood whose developing map rolls around a vertex in the plane one time for every three revolutions of the cone. Similar phenomena occur for vertices with 3 triangles or 1

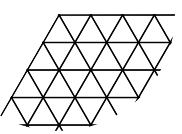
triangle.

A particularly nice phenomenon happens for any vertices that have only 1,2 , or 3 triangles. Consider a component $N_{v}$ of the inverse image in $\tilde{T}$ of a small neighborhood of any such vertex $v$. It develops into the vicinity of some vertex $w$ in Eis. In these cases, the number of triangles around $v$ is a divisor of 6 , so the developing map repeats itself when it first wraps around the vertex $w$, along a path in $\tilde{T}$ which maps to a curve in $T$ wrapping respectively 6,3 , or 2 times around the $v$. Therefore, the developing map is defined from a smaller covering of $T$ minus its singular vertices, which can be obtained as a certain quotient space $S(T)$ of $\tilde{T}$. In $S(T)$, each component of the preimage of a small neighborhood of $v$ only intersects six triangles. In fact, $S(T)$ is isomorphic to $E$. Therefore $T$ is a quotient space of a discrete group $\Gamma(T)$ acting on $E$ such that only elements of Eis are fixed points of elements of $\Gamma(T)$.

The examples where every vertex has $1,2,3$ or 6 triangles are $P(n ; 4,4,4), P(n ; 3,4,5)$ and $P(n ; 3,3,3,3)$. For $P(n ; 4,4,4)$ or $P(n ; 3,4,5)$, the group $\Gamma(T)$ is a triangle group. A fundamental domain can be chosen as the union of two equilateral triangles in the first case and $30^{\circ}, 60^{\circ}, 90^{\circ}$ triangles of opposite orientation in the second. We may arrange that one of the vertices is at the origin.

Let $\alpha$ be a singular vertex closest to the origin.

In the case $T \in P(n ; 4,4,4)$, the other singular vertices are $\mathbf{E i s} * \alpha$. Clearly this set determines the group, and any $\alpha \neq 0$ will work. The value of $n$ is the ratio $\alpha \bar{\alpha}$ of a fundamental parallelogram $0, \alpha, \alpha(1+\omega), \alpha \omega$ to the area of a

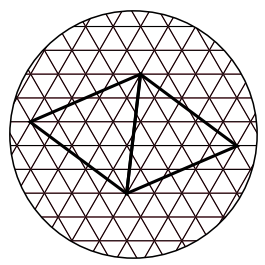
primitive lattice parallelogram $0,1,1+\omega, \omega$. The possible numbers of triangles are numbers expressible in the form $n=a^{2}+3 b^{2}$.

There is some ambiguity in this description: if we replace $\alpha$ by any of the other 5 numbers $\omega^{k} \alpha$, we obtain an isomorphic triangulation. Thus, triangulations of this type are in one-to-one correspondence with lattice points on the cone $\mathbb{C} /\langle\omega\rangle$, where $\langle\omega\rangle$ refers to the multiplicative subgroup of order 6 generated by $\omega$. 


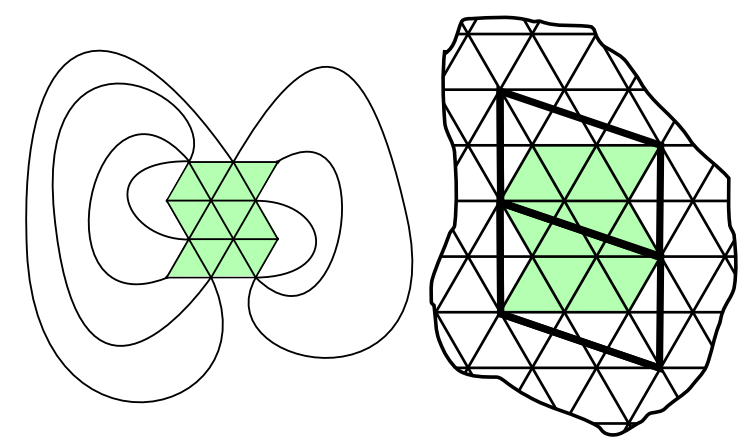

Figure 7: Developing a triangulation with 3 or 6 triangles at each vertex.

Similarly, in the case $T \in P(n ; 3,4,5)$, the vertices are of the form $(m+$ $p \sqrt{-3}) \alpha$, and $n=2 \alpha \bar{\alpha}$. As before, $\alpha$ is well-defined only up to multiplication by powers of $\omega$. In this case, if we replace $\alpha$ by $\omega^{k} \alpha$, where $k$ is odd, we get a different triangle group, but it has an isomorphic quotient space.

The case $P(n ; 3,3,3,3)$ allows somewhat more variation. For a singular vertex $x$ in Eis, let $\gamma_{x} \in \Gamma(T)$ be the rotation of order 2 about $x$. Then for any two elements $x$ and $y$, the product $\gamma_{x} \gamma_{0} \gamma_{y}$ is a $180^{\circ}$ rotation about $x+y$. Therefore, the singular vertices form an additive subgroup of Eis. Any additive subgroup will work. The subgroup is determined if we specify the sides $\alpha$ and $\beta$ of a fundamental parallelogram. If we express $\alpha$ and $\beta$ as linear combinations of the generators 1 and $\omega$ for Eis, then the value of $n$ is twice the determinant of the resulting two by two matrix. Every even number is achievable. Of course, $\alpha$ and $\beta$ are well-defined only up to change of basis for the lattice and up to multiplication by 6 th roots of unity. Note that multiplication by $\omega^{3}=-1$ is also represented by a change of generators. A nice picture can be formed by considering the shape parameter $z=\beta / \alpha$. The action of the group $S L(2, \mathbb{Z})$ on the set of shape parameters is the usual action by fractional linear transformations on the upper half plane. Figure 8 illustrates the set of shapes obtainable for $n=246$.

Let us now skip to a more complicated case, that of $P(n ; 2,2,2,2,2,2)$, which includes the regular octahedron. We have already encountered a special case: the hexagonal envelopes of section 1 are examples of octahedra of this sort.

Just as a hexagon can be described by removing three small triangles from a large triangle, there is a way to describe any element $T \in P(n ; 2,2,2,2,2,2)$ by modifying an element $\bar{T} \in P(m ; 3,3,3)$, for some $m$.

Suppose $T$ is any triangulation of the sphere with 6 vertices incident to four triangles, and the rest incident to 6 . 


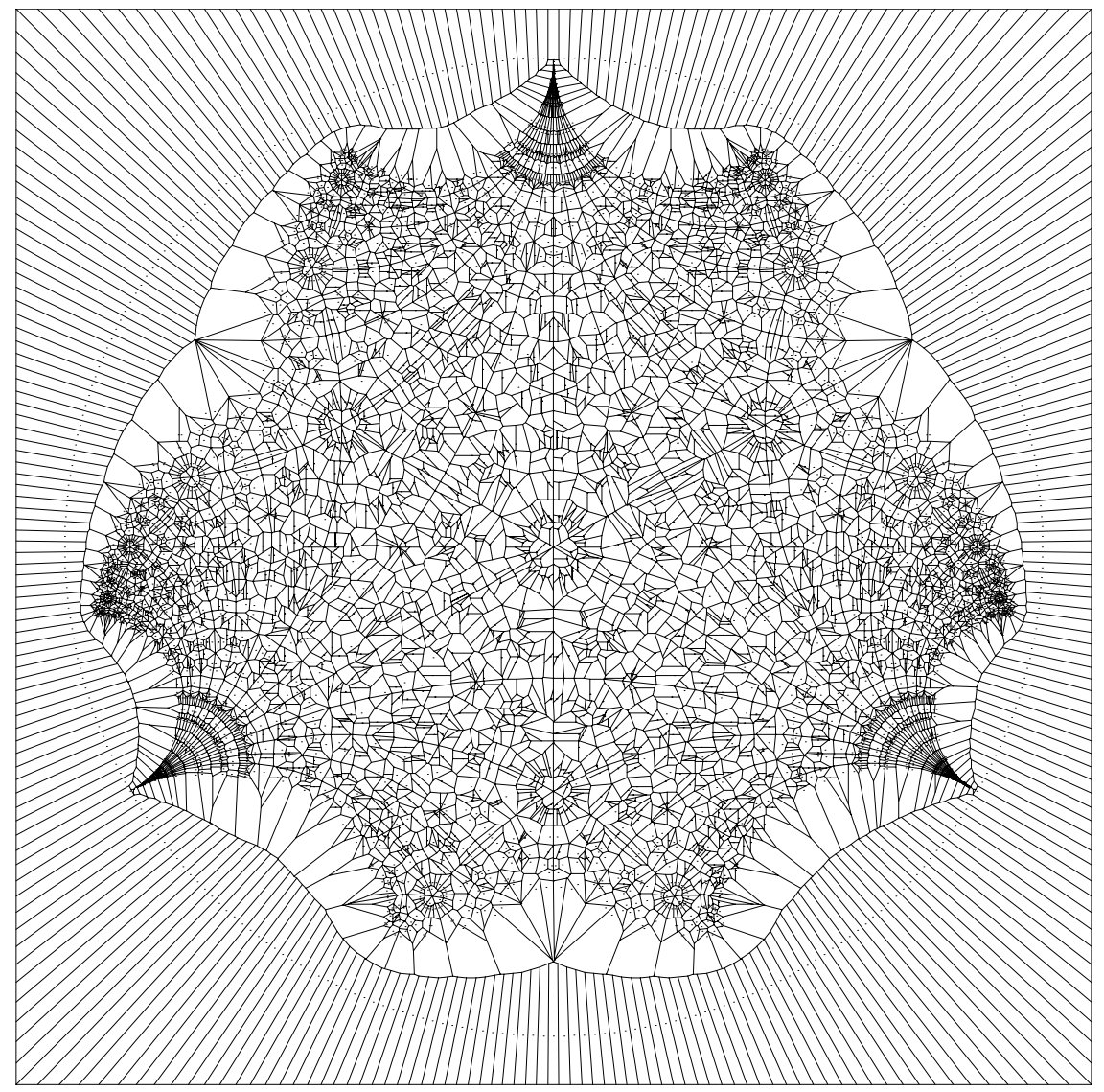

Figure 8: This is $P(246 ; 3,3,3,3)$, plotted in the Poincaré disk model of $\mathbb{H}^{2}$. The elements of $P(246 ; 3,3,3,3)$ are small dots; the Voronoi diagram for these dots is shown, with one small dot inside each region. The position of the dot in $\mathbb{H}^{2}$ determines the shape of a tetrahedron triangulated by 246 equilateral triangles. Two dots which differ by $\operatorname{PSL}(2, \mathbb{Z})$ represent the same shape. The shape does not always completely determine the triangulation - one also needs an angle for edges, that is, a lifting of the point to a certain line bundle over $\mathbb{H}^{2}$.

Consider the associated cone metric $C$. We claim there is at least one way to join the 6 cone points in pairs by three disjoint geodesic segments. To construct such a pairing, first observe that any pair of cone points are joined by at least one geodesic: the shortest path between them is a geodesic. Note that geodesics can never pass through cone points with positive curvature, except at their endpoints. We see that there is a collection of three not-necessarily disjoint geodesic segments joining the 6 points in pairs. Let $\{e, f, g\}$ be such a collection 

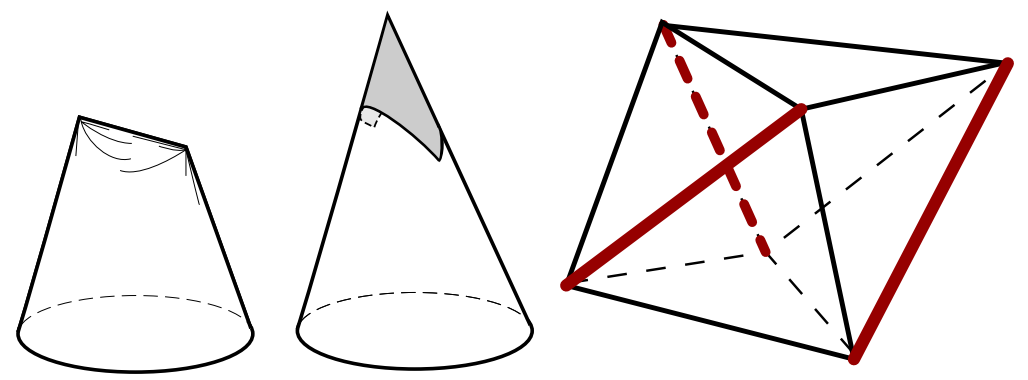

Figure 9: Left If a Euclidean cone manifold is cut along a geodesic arc joining the two cone points of curvature $\alpha$ and $\beta$, the resulting figure is isometric to a region in a Euclidean cone manifold with a new cone point whose curvature is $\alpha+\beta$ (middle). This gives a recursive procedure to reduce the construction of compact Euclidean cone manifolds of non-positive curvature to ones having only three cone points. Right An element $T \in P(n ; 2,2,2,2,2,2)$ can be reduced to $T^{\prime} \in P\left(n^{\prime} ; 3,3,3\right)$ by slitting 3 arcs, then extending.

of shortest possible length. In particular, $e, f$ and $g$ are shortest paths with their given endpoints. No pair of these edges can intersect: if they did, then by cutting and pasting, one would find that the four endpoints involved could be joined in an alternate way by shorter paths.

Cut $C$ along the three edges $e, f$ and $g$, and consider the developing map for the resulting surface $C^{\prime}$. At an endpoint of say $e$, the developing image subtends an angle of $120^{\circ}$; a curve which wraps three times around $e$ in a small neighborhood develops to a curve wrapping once around the outside of a regular hexagon $H_{e}$ in the plane. Let $C_{e}$ be $H_{e}$ modulo a rotation of order 3 . If we glue $C_{e}$ and the similarly constructed cones $C_{f}$ and $C_{g}$ to the cuts, we obtain a new cone-manifold $C^{\prime \prime}$, with three cone points of order 3 . The hexagon $H_{e}$ has its vertices on lattice points of Eis, so its center is also a lattice point of Eis. Therefore, $C^{\prime \prime} \in P(m ; 4,4,4)$ for some $m$. Consequently, a general element of $P(n ; 2,2,2,2,2,2)$ is obtained by choosing some $m$ bigger than $n$, choosing an element of $P(m ; 4,4,4)$, and choosing three types of hexagons whose area in triangles adds to $6(m-n)$ such that when they are placed around the three classes of order 3 points in the plane, all their images are disjoint. Cut all these hexagons out of the plane, divide by the $(3,3,3)$ triangle group, and glue together the pair of edges coming from each hexagon. We can express this as a choice of four elements $\alpha_{i} \in \mathbf{E i s}$, such that

$$
\alpha_{1} \bar{\alpha}_{1}-\alpha_{2} \bar{\alpha}_{2}-\alpha_{3} \bar{\alpha}_{3}-\alpha_{4} \bar{\alpha}_{4}:
$$

$\alpha_{1}$ is used to construct the original $(3,3,3)$ triangle group, and the other $\alpha_{i}$ 's are vectors from the centers of the each of the hexagons to one of the vertices, 


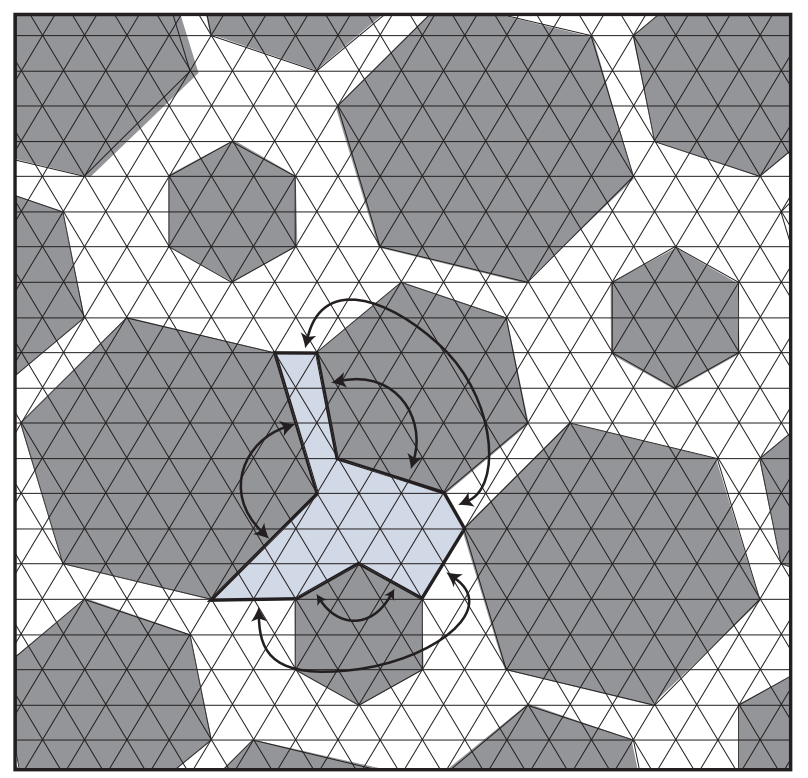

Figure 10: This is an illustration of the construction of a generalized octahedron, that is, an element of $P(n ; 2,2,2,2,2,2)$. First, choose a 3,3,3 group acting in the plane with the fixed points of the elements of order 3 on lattice points of Eis. Then choose three families of lattice hexagons invariant by the group, centered at the fixed points of elements of order 3 . Remove the hexagons, form the quotient by the group, and glue the edges of the resulting slits together. Equivalently, you can glue the boundary of a fundamental domain as illustrated.

yielding a triangulation

$$
T\left(\alpha_{1}, \alpha_{2}, \alpha_{3}, \alpha_{4}\right) \in P(n ; 2,2,2,2,2,2) .
$$

The $\alpha_{i}$ 's are subject to an additional geometric condition, that the hexagons they define be embedded. The coordinates are only defined up to a geometrically-defined equivalence relation, having to do with the multiplicity of choices for $e, f$, and $g$. The easy observation is that when any of the $\alpha_{i}$ are multiplied by powers of $\omega$, we obtain the same $T$. These coordinates make it easy to automatically enumerate all examples, although it is somewhat harder to weed out repetitions. The geometric conditions can be nearly determined from the norms: if $\left|\alpha_{i}\right|+\left|\alpha_{j}\right|<\left|\alpha_{1}\right|$, for $i \neq j \in\{2,3,4\}$, then the hexagons are disjoint; if this sum is greater than $(2 / \sqrt{3})\left|\alpha_{1}\right|=1.1547 \ldots\left|\alpha_{1}\right|$, then two hexagons intersect; otherwise, one needs to consider the picture. If $\left|\alpha_{i}\right|<\left|\alpha_{1}\right| / 3$ for $i>1$, then the three edges $e, f$ and $g$ are clearly the three shortest possible edges; in general, the question is more complicated. The standard octahedron 
$O \in P(4 ; 2,2,2,2,2,2)$, for example, has an infinite number of descriptions, for example $O=T(2 k+1+(-k+2) \omega, k+\omega, k+\omega, k+\omega)$ for every $k \geq 0$.

Another construction will be given in section 7 that can be used to search all possibilities while weeding out repetition fairly efficiently.

\section{Shapes of polyhedra}

Any collection of $n$-dimensional Euclidean polyhedra whose $(n-1)$-dimensional faces are glued together isometrically in pairs yields an example of a conemanifold and gives a pretty good flavor for the singular behavior that can occur. However, polyhedra are not a suitable substrate for a definition in the context we need, since we will be working with metrics whose local geometry has no concept of polyhedra comparable to the Euclidean case: they have no totally geodesic hypersurfaces.

In general, a cone-manifold is a kind of singular Riemannian metric; in our case, we will work with spaces modelled after a complete Riemannian $n$-manifold $X$ together with a group $G$ of isometries of $X$, called an $(X, G)$-manifold. If $G$ acts transitively, this would be called a homogeneous space, but $G$ does not necessarily act transitively. Moreover, the group $G$ is part of the structure. It is not necessarily the full group of isometries of $X$ : for instance, we might have $X=\mathbb{E}^{2}$ and $G$ the group of isometries that preserve the Eis.

An $(X, G)$-manifold is a space equipped with a covering by open sets with homeomorphisms into $X$, such that the transition maps on the overlap of any two sets is in $G$.

The concept of an $(X, G)$-cone-manifold is defined inductively by dimension, as follows:

If $X$ is 1 -dimensional, an $(X, G)$-cone-manifold is just an $(X, G)$-manifold.

Suppose $X$ is $k$-dimensional, where $k>1$. For any point $p \in X$, let $G_{p}$ be the stabilizer of $p$, and let $X_{p}$ be the set of tangent rays through $p$. Then $\left(X_{p}, G_{p}\right)$ is a model space of one lower dimension. If $Y$ is any $\left(X_{p}, G_{p}\right)$-cone-manifold, there is associated to it a fairly intuitive construction, the radius $r$ cone of $Y$, $C_{r}(Y)$ for any $r>0$ such that the exponential map at $p$ is an embedding on the ball of radius $r$ in $T_{p}(X)$, constructed from the geodesic rays from $p$ in $X$ assembled in the same way that $Y$ is. That is, for each subset of $X_{p}$, there is associated a cone in the tangent space at $p$, and to this is associated (via the 
exponential map) its radius $r$ cone in $X$. These are glued together, using local coordinates in $Y$, to form $C_{r}(Y)$.

An $(X, G)$-cone-manifold is a space such that each point has a neighborhood modelled on the cone of a compact, connected $\left(X_{p}, G_{p}\right)$-manifold.

One reason for considering inhomogeneous model spaces $(X, G)$ is that even if we start with an example as homogeneous as $\left(\mathbb{C P}^{n}, U(n)\right)$, during the inductive examination of tangent cones we soon encounter model spaces $(X, G)$ where $G$ is not transitive.

If $C$ is an $n$-dimensional $(X, G)$-cone-manifold, then a point $p \in C$ is a regular point if $p$ has a neighborhood equivalent as an $(X, G)$-space to a neighborhood in $X$, otherwise it is singular. It follows by induction that regular points are dense, and that $C$ is the metric completion of its set of regular points. The distinction between regular points and singular points can be refined to give the concept of the codimension of a point $p \in C$. If the only cone type neighborhood that a point $p$ belongs to is the neighborhood centered at $p$, then $p$ has codimension $n$. Otherwise, there is some cone neighborhood centered at a different point $q$ that $p$ belongs to, and the codimension of $p$ is defined inductively to be the codimension of the ray through $p$ in $\left(X_{q}, G_{q}\right)$.

By induction, it follows that each point $p$ of codimension $k$ is on an $(n-$ $k$ )-dimensional stratum of $C$ which is locally isometric to a totally geodesic subspace $E_{p} \subset X$ - this stratum is an $\left(E_{p}, G\left(E_{p}\right)\right)$-space, where $G\left(E_{p}\right)$ is the subgroup of $G$ sending $E_{p}$ to itself.

An oriented Euclidean, hyperbolic, or elliptic cone-manifold of dimension $n$ is a space obtained from a collection of totally geodesic simplices via a 2 to 1 isometric identification of their faces.

Suppose that $n$ numbers $\alpha_{i}$ are specified, all less than 1 , such that $\sum \alpha_{i}=2$. Let $C\left(\alpha_{1}, \alpha_{2}, \ldots, \alpha_{n}\right)$ be the space of Euclidean cone-manifold structures on the sphere with $n$ cone singularities of curvature $\alpha_{i}$ (cone angles $2 \pi\left(1-\alpha_{i}\right)$ ), up to equivalence by orientation-preserving similarity. We do not specify any homotopy class of map relative to the cone points, nor any labelling of the cone-points in these equivalences. Let $P\left(A ; \alpha_{1}, \ldots, \alpha_{n}\right)$ be the finite-sheeted covering in which the cone points can be consistently labelled. Note that the fundamental group of $P\left(A ; \alpha_{1}, \ldots, \alpha_{n}\right)$ is the pure braid group of the sphere, and the fundamental group of $C\left(\alpha_{1}, \ldots, \alpha_{n}\right)$ is contained in the full braid group of the sphere and contains the pure braid group. The exact group depends on the collection of angles, since only cone points with equal angles can be interchanged. 
How can we understand these spaces? We will first construct a local coordinate system for the space of shapes of such cone-metrics, in a neighborhood of a given metric $g$.

Proposition 3.1 (Cone-metrics have triangulations) Let $C$ be any metric on the sphere which is locally Euclidean except at isolated cone-points of positive curvature. Then $C$ admits a triangulation in the sense of a subdivision of $C$ by images of geodesic Euclidean triangles, possibly with identifications of vertices and/or edges, with vertex set the set of cone points.

Proof Associated to each cone point $v$ of $C$ is the open Voronoi region for $v$, consisting of those points $x \in C$ which are closer to $v$ than to any other cone point, and furthermore, have a unique shortest geodesic arc connecting $x$ to $v$. A Voronoi edge consists of points $x$ that have exactly two shortest geodesic arcs to cone points. Each Voronoi edge is a geodesic segment. It can happen that a Voronoi edge has the same Voronoi region on both sides if $C$ has a fairly long, skinny region with a cone point $v$ far from other cone points. Take any point $x$ on a Voronoi edge, and let $D$ be the largest metric ball centered at $x$ whose interior contains no cone points. Then $D$ is the image of an isometric immersion of a Euclidean disk $D^{\prime}$ with exactly two points $v_{1}, v_{2} \in \partial D^{\prime}$ that map to cone points of $C$. The chord $\overline{v_{1} v_{2}}$ of $D^{\prime}$ maps to an arc in $C$. The collection of all such arcs have disjoint interiors, for if not, one could lift the situation to $\mathbb{E}^{2}$ : whenever two chords of two distinct disks in $\mathbb{E}^{2}$ cross, at least one of the four endpoints is in the interior of at least one of the two disks.

The Voronoi vertices are those points that have three or more shortest arcs to cone points. The largest metric disk about a Voronoi vertex with no cone points in the interior is the image of an isometrically immersed Euclidean disk. The convex hull of the set of points on the boundary of the Euclidean disk that map to cone points is a convex polygon mapping to $C$ with boundary mapping to the edges previously constructed. Subdivide each of these polygons into triangles by adjoining diagonals. The result is a geodesic triangulation of $C$ in the sense of the proposition whose vertex set is the set of cone points.

Let $T$ be any geodesic triangulation of the cone-manifold $C$; it might or might not be obtained by this construction. Choose one of the edges of $T$, and map it isometrically into $\mathbb{C}$, with one endpoint at the origin. This map extends to an isometric developing map $D: \tilde{C} \rightarrow \mathbb{C}$, where $\tilde{C}$ is the universal cover of the complement $C_{0}$ of the vertices of $C$. Associated with each directed edge $e$ of the triangulation $\tilde{T}$ of $\tilde{C}$ is a complex number $Z(e)$ (really a vector), the 
difference between its endpoints. These vectors satisfy the cocycle condition, that the sum of the vectors associated to the oriented boundary of a triangle is 0 . Let $H: \pi_{1}\left(C_{0}\right) \rightarrow \operatorname{isom}\left(\mathbb{E}^{2}\right)$ be the holonomy of the Euclidean structure, and let $H_{0}: \pi_{1}\left(C_{0}\right) \rightarrow S^{1} \subset \mathbb{C}$ be its orthogonal part. If $\tau_{\gamma}$ is the covering transformation of $\tilde{C}$ over $C_{0}$ associated with the element $\gamma \in \pi_{1}\left(C_{0}\right)$, then $Z\left(\tau_{\gamma}(e)\right)=H_{0}(\gamma) Z(e)$. In other words, it is a cocycle with twisted coefficients - the coefficient bundle is the tangent space of $C_{0}$. Euclidean structures near $C$, up to scaling, are parametrized by cocycles near $Z$, up to multiplicative complex numbers, since any nearby cocycle determines a collection of shapes of triangles which can be glued together to form a cone-manifold with the same set of cone angles.

It is clear that change of coordinates, from those given by $T$ to those given by a triangulation $T^{\prime}$, is a linear map, since the developing map for the edges of $T^{\prime}$ can be computed as a linear function of a cocycle expressed in terms of $T$.

Proposition 3.2 (Dimension is $n-2$ ) The complex dimension of the space of cocycles, as described above, is $n-2$, where $n$ is the number of vertices.

See [8] for various computations related to this.

Proof We will describe a concrete construction for a basis for the cocycles, which amounts to making a gluing diagram to construct $C$ from a polygonal region on a cone. ${ }^{1}$

We will divide the set of edges into leaders (the basis elements) and followers. Begin by picking any vertex $v_{\text {last }}$ of $T$, and designate all edges leading into that vertex as followers. Now pick a tree in the 1 -skeleton connecting all vertices except $v_{\text {last }}$ : these will be leaders. The remaining edges are additional followers. There is a dual tree, in the dual 1-skeleton of the cell-division formed by removing the followers touching $v_{\text {last }}$, consisting of the 2 -cells and the remaining followers.

Suppose the value of a 1-cocycle is specified on each of the leaders. We can then calculate it on each of the followers, as follows. Inductively, if the current dual tree of undetermined values is bigger than a single point, pick a leaf of the tree. This is a follower which is part of a triangle whose other two sides have determined values; from them, we determine the value for the follower to

\footnotetext{
${ }^{1}$ In the general, complicated cases, this would likely be an immersed polygonal region on a cone.
} 
satisfy the coboundary condition on the given triangle. What remains is still a tree.

Finally, we are left with everything determined, except for $v_{\text {last }}$ and its remnant cluster of followers. At this point, we have enough information to determine the affine holonomy around $v_{\text {last }}$. The orthogonal part is a non-trivial rotation, so that it has a unique fixed point. The values of the cocyle for the remaining followers are determined by pointing them toward the fixed point.

A spanning tree for the $n-1$ vertices excluding the last has $n-2$ edges, so the space of cocycles is $\mathbb{C}^{n-2}$. The projective space then has dimension $n-3$.

The area of a cone-manifold structure defines a hermitian form on the space of cocyles: that is, given a cocycle $Z, A(Z)=\frac{1}{4} \sum_{\text {triangles }} i e_{1} \bar{e}_{2}-i e_{2} \bar{e}_{1}$ where in local coordinates $e_{1}$ and $e_{2}$ are successive edges of the triangle proceeding counterclockwise. Obviously $A(Z)$ is independent of choice of local coordinates.

Proposition 3.3 (Signature $(1, n-3)$ ) If each of the $\alpha_{i}>0$, then $A$ is a hermitian form of signature $(1, n-3)$.

Proof We have seen this illustrated in several examples already. There is a general procedure for diagonalizing the expression for area. If $C$ has only three vertices, then the vector space is only one dimensional, so $A$ is necessarily positive definite: it is proportional to the square of the length of any of the edges of $T$.

We have already seen the special case that there are four cone angles all equal to $\pi$, under the guise of $P(n ; 3,3,3,3)$. The expression for area is the determinant of a $2 \times 2$ real matrix, made of the real and imaginary parts of two of the values $Z(e)$. Since determinants can be positive or negative, this is a hermitian form of signature $(1,1)$.

In every other case, there are at least two cone angles whose curvatures have sum less than $2 \pi$. Construct any geodesic path $e$ between them, slit $C$ open, and glue a portion of a cone with curvature the sum of the two curvatures to obtain a cone-manifold $C^{\prime}$ with one fewer singular points (figure 9). The area of $C$ is the area of $C^{\prime}$ minus a constant times the square of the length of $e$. This gives an inductive procedure for diagonalizing $A$, inductively showing that the signature of the area is $(1, n-3)$.

The set of positive vectors in a Hermitian form of signature $(1, n-3)$ up to multiplication by scalars, is biholomorphic to the interior of the unit ball in 
$\mathbb{C}^{n-3}$, and is known as complex hyperbolic space $\mathbb{C H}^{n-3}$. A metric of negative curvature is induced from the Hermitian form; as a Riemannian metric, its sectional curvatures are pinched between -4 and -1 . Therefore, $C\left(A ; \alpha_{1}, \ldots, \alpha_{n}\right)$ is a complex hyperbolic manifold.

It is not metrically complete, however. Any two singular points of a $c$ whose curvature adds to less than $2 \pi$ can collide as the cone-metric changes a finite amount, measured in the complex hyperbolic metric. We will next examine how to adjoin to $C\left(\alpha_{1}, \ldots, \alpha_{n}\right)$ the degenerate cases where one or more of the cone points collide, to obtain a space $\bar{C}\left(\alpha_{1}, \ldots, \alpha_{n}\right)$ which is the metric completion of $C\left(\alpha_{1}, \ldots, \alpha_{n}\right)$.

Each element $c$ of $\bar{C}\left(\alpha_{1}, \ldots, \alpha_{n}\right)$ is associated with some partition $P$ of the angles $\alpha_{i} ; c$ is a Euclidean cone-manifold where each cone point is associated with a partition element $p \in P$ and has curvature equal to the sum of the elements of $p$. We regard two partitions as equivalent if one can be transformed to the other by a permutation of the index set which preserves the values of the $\alpha_{i}$. A limit of a sequence of cone-manifolds associated with some partition will be associated with a coarser partition, if distances between some of the cone points in the sequence tend to zero.

Theorem 3.4 (Completion is cone-manifold) The metric completion of $C\left(A, \alpha_{1}, \ldots, \alpha_{n}\right)$ is $\bar{C}\left(\alpha_{1}, \ldots, \alpha_{n}\right)$, which is a complex hyperbolic cone-manifold.

Proof There is a very natural way to describe regular neighborhoods for the stratum $S_{P}$ corresponding to a partition $P$ of the set of curvatures concentrated at cone points.

Consider an element $c \in C\left(\alpha_{1}, \ldots, \alpha_{n}\right)$ such that the cone points are clustered in accordance with $P$. We may assume that the diameter of each cluster is less than the minimum distance from the cluster to any cone point not in the cluster, and less than some small constant $\epsilon$.

The holonomy for a curve which goes around any cluster $D$ is a rotation by the total curvature of $D$, unless the total curvature is $2 \pi$. When the total curvature of $D$ is $2 \pi$, the holonomy is a translation. If the holonomy is actually a rotation, it leaves invariant each of a family of circles; with our assumption that the cluster is isolated from other cone points, the encircling curve is isotopic to one of these circles.

If the total curvature of $K(D)$ is less than $2 \pi$, the surface of $c$ near such a circle isometrically matches a cone with apex on the same side as the cluster, with 
cone point of curvature $K(S)$. In this case, we can define a new cone-manifold $p(c)$ by cutting out each cluster, and replacing it by a portion of this cone. In local coordinates, this gives a local orthogonal projection from a neighborhood of $c$ to $S_{P}$. The distance from the singular stratum is $\sqrt{\operatorname{area} p(c)-\operatorname{area} c}$. Note that the normal fibers for strata corresponding to subclusters of a cluster are contained in normal fibers for the larger cluster.

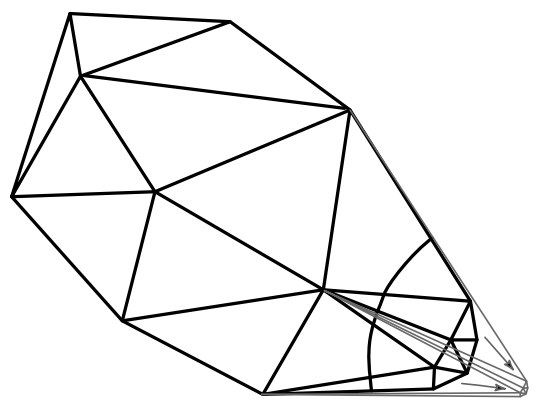

Figure 11: Any cluster of cone points close together compared to the distance to other cone points can be shrunk to a single cone point. This process gives a radial structure to a neighborhood of a singular point in the space of cone-metrics with designated curvatures on a sphere.

The total curvature cannot be greater than $2 \pi$, if $\epsilon$ is chosen properly: in that case, $c$ would match the surface of a cone with apex on the opposite side from the cluster. The area of $C$ is less than the area of the portion of cone, plus the area within the cluster, so that if $\epsilon$ is small compared to $\theta / A$, where $\theta$ is the minimum value by which a curvature sum can exceed $2 \pi$, this cannot occur.

A cluster of arbitrarily small diameter with total curvature $2 \pi$ can occur, but this forces the diameter of $c$ to be large: in this case, $c$ matches the surface of a cylinder outside a neighborhood of the cluster, and there is a complementary cluster at the other end of the cylinder. As $c$ moves a finite distance in the complex hyperbolic metric, its diameter cannot go to infinity, so no such cluster goes to 0 in diameter in the metric completion of $C\left(\alpha_{1}, \ldots, \alpha_{n}\right)$.

Within any bounded set of $C\left(\alpha_{1}, \ldots, \alpha_{n}\right)$, we are left only with the case of small diameter clusters whose total curvature is less than $2 \pi$.

It is now easy to see that $\bar{C}\left(\alpha_{1}, \ldots, \alpha_{n}\right)$ is the metric completion of $C\left(\alpha_{1}, \ldots, \alpha_{n}\right)$ and that it is a complex hyperbolic cone-manifold.

Of particular importance are the strata of complex codimension 1 or real codimension 2. These strata correspond to the cases when only two cone points of $c$ have collided. What are the cone angles around these strata?

Proposition 3.5 (Cone angles around collisions) Let $S$ be a stratum of $C\left(\alpha_{1}, \ldots, \alpha_{n}\right)$ where two cone points with curvature $\alpha_{i}$ and $\alpha_{j}$ collide.

If $\alpha_{i}=\alpha_{j}$, the cone angle $\gamma(S)$ around $S$ is $\pi-\alpha_{i}$, otherwise it is $2 \pi-\alpha_{i}-\alpha_{j}$. 
In other words, the cone angle in parameter space is the same as the physical angle two nearby cone points go through, as measured from the apex of the cone that would be formed by their collapse, when they revolve about each other until they return to their original arrangement.

Proof When cone points $x_{i}$ and $x_{j}$ with these two angles are close together on a cone manifold $c$, we can think of $c$ as constructed from $p(c)$ by replacing a small neighborhood of the cone by a portion of a modified cone $D\left(\alpha_{i}, \alpha_{j}\right)$ with two cone points. The shape of $D\left(\alpha_{i}, \alpha_{j}\right)$ is uniquely determined by $\alpha_{i}$ and $\alpha_{j}$ up to similarity. Thus, the shape of $c$ is determined by selecting the point $x_{i}$ on $p(c)$, and may be represented by $p(c)$ together with the vector $V$ from the combined cone point of $p(c)$ to $x_{i}$. In local inhomogeneous coordinates coming from a choice of a triangulation, $V$ is a locally linear function, described by a single complex number.

If $\alpha_{i}=\alpha_{j}$, then when the argument of $V$ is increased by half the cone angle, or $\pi-\alpha_{i}, x_{i}$ and $x_{j}$ are interchanged, and the resulting configuration is indistinguishable. Therefore, $\pi-\alpha_{i}$ is the cone angle along $S$, (and $\pi+\alpha_{i}$ is the curvature concentrated at $S$ ). If $\alpha_{i} \neq \alpha_{j}$, the argument of $V$ must be increased by the cone angle, $2 \pi-\alpha_{i}-\alpha_{j}$, before the same configuration is obtained again. In this case, $2 \pi-\alpha_{i}-\alpha_{j}$ is the cone angle along $S$, and $\alpha_{i}+\alpha_{j}$ is the curvature concentrated along $S$.

More generally, if $S$ is a stratum of complex codimension $j$ representing the collapse of a cluster of $j+1$ cone points, each normal fiber to $S$ is a union of 'complex rays', swept out by an ordinary real ray by rotating it the direction $i$ times the radial direction. The space of complex rays is the complex link of the stratum, a complex cone-manifold whose complex dimension is one lower. The real link is a Seifert fiber space over the complex link, with generic fiber a circle of length $\alpha$ which we can call the scalar cone angle $\gamma(S)$ at $S$. We define the real link fraction of $S$ to be the ratio of the volume of the real link of $S$ to the volume of $S^{2 j-1}$ (the real link in the non-singular case), and similarly the complex link fraction is the ratio of the volume of the complex link to the volume of $\mathbb{C P}^{j-1}$.

Proposition 3.6 (Cone angles for multi-collisions) Let $S$ be a stratum of complex codimension $j$ where $j+1$ cone points of curvature $\kappa_{1}, \ldots, \kappa_{j}$ collapse.

Let $N$ be the order of the subgroup of the symmetric group $S_{j}$ that preserves these numbers. Then: 
a) The scalar cone angle is

$$
\gamma(S)=2 \pi-\sum_{i} \kappa_{i}
$$

b) The complex link fraction is

$$
\frac{(\gamma(S) / 2 \pi)^{j-1}}{N}
$$

c) The real link fraction is

$$
\frac{(\gamma(S) / 2 \pi)^{j}}{N}
$$

Proof The proof of part (a) is the same as above, with the observation that a cluster of 3 or more cone points can always be slightly perturbed to make it asymmetrical, so in the generic fiber of the Seifert fibration (obtained by rotating the cluster of cone points) no permutations of the cone points occur.

For (b), think first about the case that all cone angles are different, so as to avoid a symmetry group at first. A neighborhood of $S$ is then a manifold, isomorphic to the limiting case when $\kappa_{i} \rightarrow 0$, the space of $(j+1)$-tuples in the plane up to affine transformations. The complex link is a complex cone-manifold structure on $\mathbb{C P}^{j-1}$. If $\omega$ is a closed 2 -form on $\mathbb{C P}^{j-1}$ that integrates to 1 over $\mathbb{C P}^{1}$, then $\omega^{j-1}$ gives the fundamental class for $\mathbb{C P}^{j-1}$. (This calculus works readily for cone metrics with differential forms that are suitably continuous.) We conveniently obtain such a form as some constant multiple $\alpha$ of the Kähler form of the model geometry $\mathbb{C P}^{j-1}$ of the link. One way to determine $\alpha$ is to reduce to the case $j=2$ by clustering the cone points into three groups which are collapsed along a codimension 2 stratum limiting to $S$. In the case $j=2$, the complex link is $S^{2}$ with cone points of curvature $\kappa_{1}+\kappa_{2}, \kappa_{2}+\kappa_{3}$ and $\kappa_{3}+\kappa_{1}$. This uses up $2 \sum \kappa_{i}$ out of the total curvature $4 \pi$ of $S^{2}$, so the area of a constant curvature metric is reduced by a factor of $\gamma(S)$.

Part (c) follows from (a) and (b), since the real link fraction is the product of the complex link fraction with $\gamma(S) / 2 \pi$.

The case with symmetry follows by dividing the asymmetric configuration space by the symmetry.

\section{Orbifolds}

An orbifold is a space locally modelled on $\mathbb{R}^{n}$ modulo finite groups; the groups vary from point to point. For an exposition of the basic theory of orbifolds, see 
[11]. Our orbifolds will be $(X, G)$-orbifolds, locally modelled on a homogeneous space $X$ with a Lie Group $G$ of isometries. It is easily seen by induction on dimension that an orientable $(X, G)$-orbifold has an induced metric which makes it into a cone-manifold. (Use the naturality of the exponential map.)

Here is a basic fact about the relation between cone-manifolds and orbifolds, which essentially is a rephrasing of Poincaré's theorem on fundamental domains:

Theorem 4.1 (Codimension 2 conditions suffice) Let $C$ be an $(X, G)$-conemanifold. Then $C$ is a "weakening" of the structure of an orbifold if and only if all the codimension 2 strata of $C$ have cone angles which that are integral divisors of $2 \pi$.

Proof An orientation-preserving group of isometries whose fixed point set has codimension 2 is a subgroup of $S O(2)$, and the only possibilities are $\mathbb{Z} / n$. The cone angle along such a stratum in an orbifold is therefore an integral divisor of $2 \pi$, and the condition is necessary.

The converse can be proved by induction on the codimension of the singular strata of $C$. Clearly, it works for strata of codimension 2. Suppose that we have proven that $C$ has an orbifold structure in the neighborhood of all strata up through codimension $k$. Let $S$ be a singular stratum of codimension $k+1$, and consider the neighborhood $U$ of a point $x \in S$. This neighborhood can be taken to have the form of a bundle over a neighborhood of $x$ in $S$, with fiber the cone on a $k$-dimensional cone-manifold $N$, the normal sphere to $S$. The normal sphere $N$ is modelled on $\left(S^{k}, G\right)$, where $G \subset S O(n)$. By induction, $N$ is an orbifold; its universal cover must be $S^{k}$, since for $k \geq 2$ the sphere is simply-connected. Therefore the cone on $N$ is the quotient of $B^{k+1}$ by the group of covering transformations of $S^{k}$ over $N$, and therefore $U$ is also the quotient space of a neighborhood in $X$ by the same group. Thus, $C$ is an orbifold.

To illustrate, let's look at some of the local orbifold structures that arise in multi-way collisions. When $k$ cone points of equal curvature $2 \pi \alpha$ collide, the order of the local group $\Gamma(S)$ for a stratum $S$ is the reciprocal of the real volume fraction, so from 3.6, setting $\alpha=\gamma(S) / 2 \pi$ we have

$$
\# \Gamma(S)=\frac{k !}{(1-k \alpha)^{k-1}} \quad\left[\frac{1}{1 / 2-\alpha} \in \mathbb{Z} \quad \& \quad 0<\alpha<1 / k\right]
$$

The only three cases satisfying the condition for three colliding equal angle cone points are when $\alpha$ is $1 / 6,1 / 4$ and $3 / 10$. The complex links in these 
three cases are the quotient orbifolds of the sphere by the oriented symmetries of one of the regular polyhedra: $(2,3,3),(2,3,4)$ or $(2,3,5)$. The real link is $S^{3}$ with a cone axis along the trefoil knot of order 3,4 or 5 . The formula gives orders for these groups of 24, 96 and 600 . (This can be quickly confirmed by an automated check using the 3-dimensional topology program Snappea to obtain presentations for the orbifold fundamental groups and feeding then to a group theory program such as Magnus.)

An interesting example of a collision of cone points of unequal curvatures is $(19 \pi / 30,11 \pi / 30,29 \pi / 30)$. The real link is an orbifold with $(2,3,5)$ cone axes on the 3 -component Hopf link. In this case, $\alpha=1 / 60$ and $\#(\Gamma(S))=(60)^{2}=$ 3600 .

The biggest possible multiple collision is when 5 points of curvature $\pi / 3$ collide. The local group for this collision has order $6^{4} 5 !=155,520$.

Infinitely many of the modular spaces for cone-metrics with 4 cone points are orbifolds of complex dimension 1, but for higher dimensional modular spaces, only 94 are orbifolds. These are tabulated in the appendix.

\section{Proof of main theorem}

Proof of Theorem 0.2 Most of this theorem follows formally from Theorem 3.4, Proposition 3.5, and Theorem 4.1. What still remains is a discussion of the volume of $\bar{C}\left(\alpha_{1}, \ldots, \alpha_{k}\right)$.

The only case in which $X=\bar{C}\left(\alpha_{1}, \ldots, \alpha_{k}\right)$ is not compact is where there are cone-manifolds $x \in X$ whose diameters tend to infinity. In such a case, if we normalize so that the area of $x$ is 1 , there must be subsets of $x$ with large diameter and small area, free from cone points. This implies that $x$ has subsets which are approximately isometric to a thin Euclidean cylinder. If $\gamma \subset x$ is a short curve going around such an approximate cylinder, then the angle of rotation for $\gamma$ must be a sum of a subset of the $\left\{\alpha_{i}\right\}$. There are only a finite number of possibilities, so if the diameter is large enough, a neighborhood of $\gamma$

of large diameter is actually a cylinder. Once $\gamma$ is determined, the shapes of the two pieces of $x$ cut by $\gamma$ can be specified independently, and a scale factor length $(\gamma)^{2} /$ area (less than some constant $\epsilon$ ) together with an angle of rotation can also be specified independently.

It will follow that the ends of $x$ are in 1-1 correspondence with partitions $Q$ of the set of curvatures into two subsets each summing to $2 \pi$, if we verify two points: 
(i) for any such partition $Q$, there exists an $x \in X$ with a geodesic $\gamma$ separating the cone points according to $Q$, and

(ii) the subspace $X_{\gamma, \epsilon}$ consisting of cone-manifolds in $X$ with a geodesic $\gamma$ of length $\epsilon$ which separates the cone points according to $Q$ is connected.

Actually, the proof does not logically depend on either point, and it is a slight digression to prove them, but it seems worth doing anyway.

An easy demonstration of (i) is to construct a polygon with angles $\pi-\alpha_{i} / 2$. It is easy to find a very thin polygon realizing $Q$. Doubling such a polygon gives a suitable cone-manifold $x$.

We will describe an explicit construction for (ii). Let us begin with the special case of $c \in X_{\gamma, \epsilon}$ which are obtained by doubling a convex Euclidean polygon whose angles are half the cone angles for $X$. It is easy to connect any two convex polygons with the same sequence of angles by a family of polygons having the same angles. If we allow degenerate cases as well, where two angles coincide, the order is irrelevant. Therefore, this special subspace of $X_{\gamma, \epsilon}$ is connected.

Therefore, it suffices to connect any $c \in X_{\gamma, \epsilon}$ to something obtained by doubling a convex polygon. Construct a maximal cylindrical neighborhood $N_{1}$ of $\gamma$ with geodesic boundary. There is at least one cone point on each boundary component of $N_{1}$. Let $\beta$ be one of the boundary components, and $x_{1} \in \beta$ a cone point, with curvature $\alpha$. If $c$ is cut along $\beta$, the portion on the other side of $\beta$ from $N_{1}$ has boundary consisting of a geodesic with a convex angle of $\pi-\alpha$ at $x_{1}$, and possibly additional angles if it contains other cone points. There is a circular arc $\beta^{\prime}$ through $x_{1}$, contained in $N$, which appears to have a convex angle of $\pi-\alpha$ from within $N$, but appears to be smooth at $x_{1}$ when viewed from the outside. Let $U_{1}$ be the "outside" component obtained by cutting along $\beta^{\prime}$. Its boundary is now locally isometric to a circle, and a neighborhood, like on a cone, is foliated by parallel circles.

Deform $c$, by shrinking the "interesting part" of $U_{1}$ relative to the rest of $c$, so that the next cone point in $U_{1}$ is not close to $\beta^{\prime}$. Let $N_{2}$ be a maximal neighborhood of $\beta^{\prime}$ which is foliated by parallel circles, and let $x_{2}$ be a point on its boundary. Adjust by a rotation of $U_{1}$ until the geodesic through $x_{1}$ perpendicular to the foliation by circles hits at $x_{2}$. Draw a circular arc through $x_{2}$, within $U_{1}$, which appears smooth from the outside neighborhood $U_{2}$.

This process can be continued, in the same manner, until the last neighborhood $U_{k}$ is a cone. The geodesic through $x_{k-1}$ automatically hits the cone point. Now do the same process on the other side of $N_{1}$, first adjusting by a rotation so a 
geodesic through $x_{1}$ perpendicular to the foliation of $N_{1}$ by parallel circles hits at a cone point.

After this sequence of deformations, we have a cone-manifold with a geodesic Hamiltonian path through all the cone points, such that at cone points internal to it the two outgoing geodesics bisect the cone angle. The path can be completed to a curve by one additional geodesic (this is easy to see if you draw the figure in the plane obtained by cutting along the path; it is made of two convex arcs, and has bilateral symmetry).

Note that a similar process works for a general cone-manifold: we do not really need $\gamma$ for this construction, we can begin at any cone point, and work outward from it.

We call the ends of $X$ cusps, in accordance with terminology for manifolds and orbifolds. To justify this word, note that each cusp is foliated by complex geodesics with respect to the Hermitian metric, obtained by rotating the two ends of $c$ with respect to each other and by scaling. The complex geodesics are locally isometric to the hyperbolic plane. The pure scaling, which may be thought of as inserting extra lengths of cylinder between the two ends, generates a real geodesic. These real geodesics converge, as the shrinking increases. The convergence is exponential, so the total volume of each cusp is finite.

\section{The icosahedron and other polyhedra}

Let $A$ be the subgroup of isometries of $\mathbb{C}$ which take Eis into itself. We may think of the classes of triangulations $P\left(n ; k_{1}, \ldots, k_{m}\right)$ as the space of $\left(\mathbb{E}^{2}, A\right)$ cone-manifolds of area $n$ (measured in double triangles) and cone angles $k_{i} \pi / 3$. They consist of elements of $C\left(k_{1} \pi / 3, \ldots, k_{n} \pi / 3\right)$ equipped with a reduction of the $\left(\mathbb{E}^{2}\right.$, isom $\left.\left(\mathbb{E}^{2}\right)\right)$ structure to $\left(\mathbb{E}^{2}, A\right)$. In more concrete terms, a triangulation is given by a cocycle whose coefficients are elements of Eis.

Euclidean cone-manifolds sometimes admit several inequivalent reductions to $\left(\mathbb{E}^{2}, A\right)$-in other words, there are some cone-manifolds that can be subdivided into unit equilateral triangles in more than one way. In complex Lorentz space $\mathbb{C}^{(m-3,1)}$, the set of cocyles with a certain total area form a sheet of a hyperboloid. The hyperboloid fibers over complex hyperbolic space, with fiber a circle (corresponding to multiplication of the cocycle by elements of the unit circle). The set of triangulations are lattice points in $\mathbb{C}^{(m-3,1)}$, and the value of the Hermitian form counts the number of triangles - multiple unit equilateral triangulations of a Euclidean manifold correspond to fibers that intersect more 
than lattice points. (All lattice points come in groups of 6 whose ratios are units in the ring Eis.)

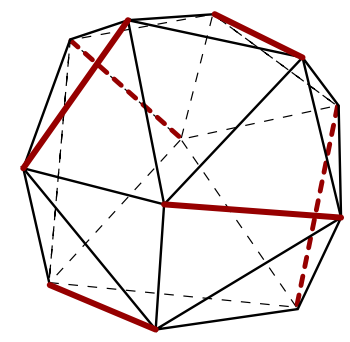

Figure 12: If an icosahedron is slit along 6 disjoint arcs joining its vertices in pairs, conical caps can be inserted to turn it into an octahedron.

The "biggest" of the classes of triangulations is

$$
P(n ; 1,1, \ldots, 1) \subset J=C(\pi / 3, \pi / 3, \ldots, \pi / 3),
$$

the one which contains the icosahedraon. The "completion" $\bar{P}(n ; 1, \ldots, 1) \subset \bar{J}$ which includes degenerate cases contains all the other classes of triangulations.

By theorem 1.2, $\bar{J}$ is a complex hyperbolic orbifold of dimension 9 . The cone angles around the complex codimension 1 singular strata are $2 \pi / 3$.

There is a concrete construction to describe an arbitrary element of $\bar{J}$ or of $\bar{P}(1, \ldots, 1)$, as follows. Suppose first that $x \in J$ is an arbitrary Euclidean cone-metric on the sphere with all cone points having curvature $\pi / 3$. Choose a collection of 6 disjoint geodesic arcs with endpoints on the cone points. Slit along each of these arcs.

Locally near the endpoints of the arcs, the developing map maps the slit surface to the complement of a $60^{\circ}$ angle. A neighborhood of the slit develops to a region outside an equilateral triangle in the plane; when you go once around the slit, the developing image goes $2 / 3$ of the way around the triangle.

For each slit, take $2 / 3$ of an equilateral triangle with side equal to the length of the slit, fold it together to form a cone point in the center of the original triangle with curvature $2 \pi / 3$ and glue it into the slit. The result is a cone-manifold $f(x)$ like the octahedron, in $C(2 \pi / 3,2 \pi / 3,2 \pi / 3,2 \pi / 3,2 \pi / 3,2 \pi / 3)$.

As in section 2, we can analyze the shape of $f(x)$ by joining its cone points in pairs by disjoint geodesic segments, slitting open, and extending to give an element of $C(4 \pi / 3,4 \pi / 3,4 \pi / 3)$ (which is a single point).

If $x \in \bar{J}-J$, the analysis still works: treat the cone points as cone points with multiplicity, and use zero-length slits as much as possible at cone points with curvature greater than $\pi / 3$. At the first step, the slits of positive length pair the cone points with curvature an odd multiple of $\pi / 3$. When the slits are filled 
in, the curvature at each of the endpoints is decreased by $\pi / 3$, and the resulting cone-manifold has all curvatures an even multiple of $\pi / 3$. For the second step, note that no cone point can have curvature $6 \pi / 3$ or bigger. In this case, the slits of positive length join cone points with curvature $2 \pi / 3$.

An arbitrary $x \in \bar{J}$ can be reconstructed by reversing this procedure.
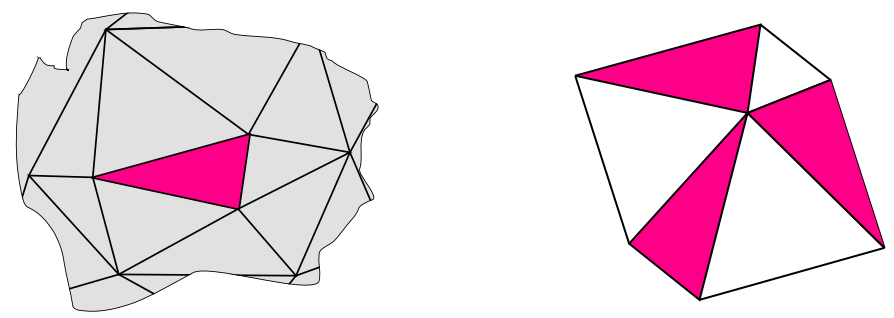

Figure 13: A geodesic triangle whose vertices are on cone points of curvature $\pi / 3$ has a deleted neighborhood that develops to the deleted neighborhood of a Napoleon hexagon, formed from three copies of the triangle and three equilateral triangles. This process, applied to the 12 vertices of an icosahedron-like cone-manifold grouped into 3 's, recursively reduces it to a tetrahedron-like cone-manifold

There are many alternative coordinate systems for $J$. For example, another construction is to group the cone points in 3 's, by constructing 4 disjoint geodesic triangles with vertices at cone points. If these triangles are cut out, then the developing image of what is left is discrete; it comes from a 2,2,2,2 group acting in the Euclidean plane. The developing image is the complement of a certain union of hexagons about the lattice of elliptic points. The hexagons are not arbitrary, however - the hexagons $H(T)$ that arise are hexagons that come from Napoleon's theorem, constructed as follows: Suppose $T$ has sides $a, b$, and $c$, in counterclockwise order. We will construct 6 triangles around the vertex $v$ of $T$ between $a$ and $b$. First construct an equilateral triangle on side $a$. Construct another triangle $T_{1}$ congruent to $T$ on the free side of the equilateral triangle which is incident to $v$. Side $c$ of $T_{1}$ also touches $v$; on this, construct a second equilateral triangle. Continue alternating copies of $T$ and equilateral triangles until it closes, yielding $H(T)$.

Note that $H(T)$ has sides $a, b, c, a, b, c$ in counterclockwise order. The complement of $H(T)$ modulo a rotation of $180^{\circ}$ has boundary which matches the boundary of $T$; when it is glued in, three cone points of curvature $\pi / 3$ are obtained at the vertices of $T$.

A general $x \in \bar{J}$ can be obtained by choosing first a 2,2,2,2 group, and then choosing four hexagons $H\left(T_{i}\right)$ centered about the four classes of vertices. Form 
the quotient of the complement of the hexagons by the group, and glue in the triangles $T_{i}$. If the hexagons are disjoint and nondegenerate, $x \in J$.

From this concrete point of view, what is amazing is that these coordinate systems have a global meaning, since $\bar{J}$ is an orbifold: even if one chooses a collection of hexagons $H\left(T_{i}\right)$ which overlap, they determine a unique Euclidean cone-manifold, provided the net area (computed formally) is positive.

Using these constructions, it is not hard to show that $P(n ; 1, \ldots, 1)$ contains 1 or more elements for all values of $n$ starting with 10 , with 11 as the sole exception. If there were an element $T$ of $P(11,1, \ldots, 1)$, it would have 13 vertices and 22 triangles. One could then construct a spherical cone-manifold by using equilateral spherical triangles with angles $2 \pi / 5$. This cone-manifold would have only one cone point — which is manifestly impossible, since the holonomy for a curve going around the cone point is a rotation of order 5 , but at the same time the holonomy is trivial since the curve is the boundary of a disk having a spherical structure.

From the picture in $C^{(1,9)}$, it follows that the number of non-negatively curved triangulations having up to $2 n$ triangles is roughly proportional to the volume of the intersection of some cone with the ball of radius $\sqrt{n}$ in this indefinite metric. The cone in question is neither compact nor convex, but since it comes from a fundamental domain for the group action, its intersection with the ball of norm less than any constant has finite 10-real-dimensional volume. Therefore, the number of triangulations with up to $2 n$ triangles is $O\left(n^{10}\right)$.

\section{$7 \quad$ An explicit construction and fundamental domain}

Another method for constructing, manipulating and analyzing non-negatively curved cone structures goes as follows:

Given $k+1$ real numbers $\alpha_{0}, \alpha_{1}, \ldots, \alpha_{k} \geq 0$ whose sum is $4 \pi$.

To Construct Euclidean cone-metrics with the $\alpha_{i}$ as curvatures.

Choose a $k$-gon $P$ in the plane, with edges $e_{1} \ldots, e_{k}$.

Construct $(i=1, \ldots k)$ : An isosceles triangles $T_{i}$ with base on $e_{i}$, apex $v_{i}$, apex angle $\alpha_{i}$, pointing inward if $\alpha_{i}<\pi$, pointing outward if $\alpha_{i}>\pi$.

Condition $\mathbf{A}$ the triangles $T_{i}$ are disjoint from each other and disjoint from $P$ except along $e_{i}$.

Let $Q$ be thefilled polygon obtained from $P$ by replacing each $e_{i}$ by the other two sides $f_{i}$ and $g_{i}$ of $T_{i}$. 
Glue $f_{i}$ to $g_{i}$ to obtain a cone manifold. The vertex $v_{i}$ becomes a cone point of curvature $\alpha_{i}$. The other $k$ vertices of $P$ all join to form a cone point of cone angle $\alpha_{0}$.

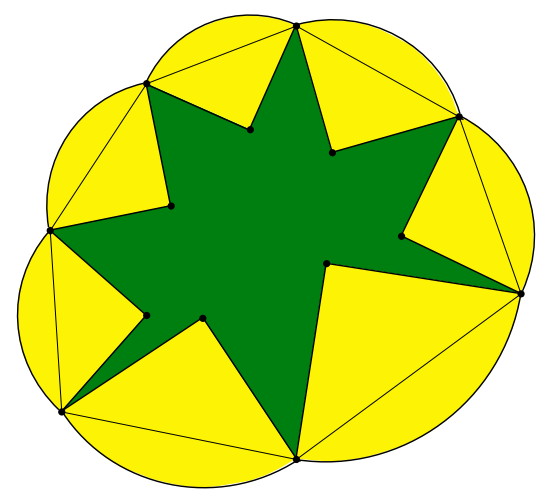

Figure 14: A cube-like cone-metric ( 8 cone-angles of curvature $\pi / 2$ ) can be constructed by removing isosceles right triangles from the sides of a heptagon and gluing the resulting pairs of equal sides. The seven sharp angles all come together to form the eigth cone point. This illustration (along with the others in this section) was constructed with the program Geometer's Sketchpad, where the shape can be varied while preserving the correct geometric relations.

As examples, see figure 14 for a cube-like cone-manifold, or figure 15 for a triangulation of $S^{2}$ with 23 vertices and 42 triangles constructed from an icosahedrallike cone-manifold.

Here is the inverse construction. Given a cone-metric with $n$ cone points on $S^{2}$ :

Choose one of the cone points $v_{0}$.

Find for each other cone point $v_{i}$ a shortest path $a_{i}$ from $v_{0}$ to $v_{i}$. The $a_{i}$ are necessarily simple and disjoint, except at $v_{0}$.

Cut along all these paths, to obtain a disk equipped with a Euclidean metric whose boundary is composed of $2(n-1)$ straight segments, each paired to an adjacent segment of the same length and forming an angle equal to the corresponding cone angle. (See figure 16.)

We will show that if $P$ is a cone-metric on the sphere with positive curvature at each vertex, and if $S(P)$ ( $S$ because it resembles a star) is the metric on $D^{2}$ obtained by cutting $P$ open as above, then $S(P)$ can be flattened out into the plane, that is, it is isometric to the metric of a filled simple polygon. 


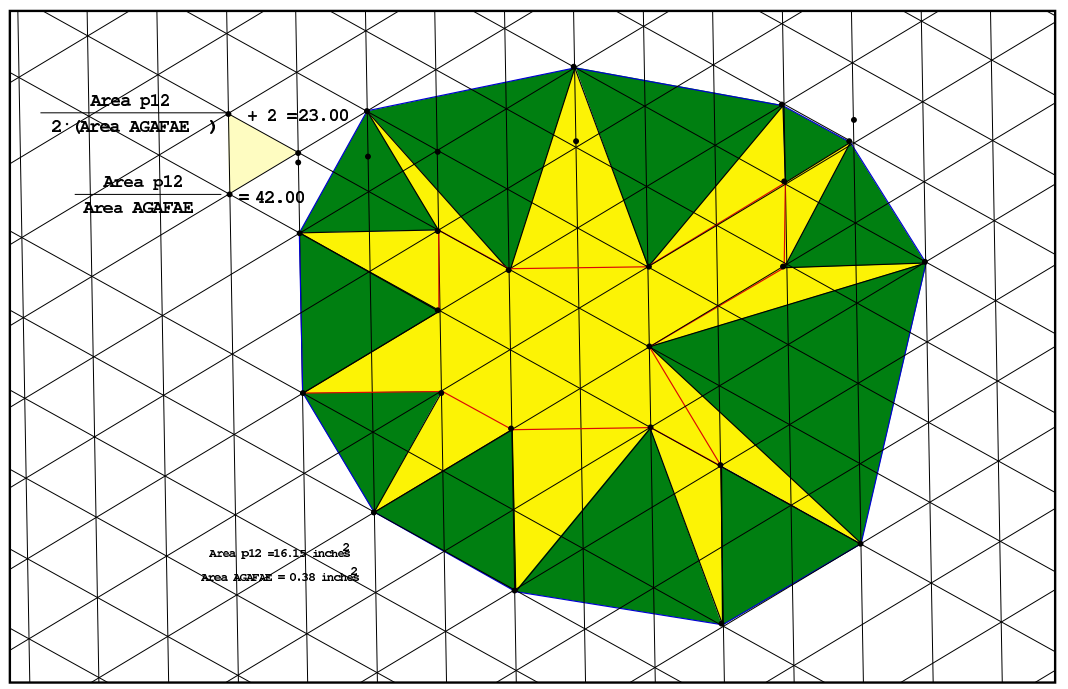

Figure 15: This is a diagram for a triangulation of $S^{2}$ with 42 triangles, having 12 vertices of order 5 and 11 vertices of order 6 . Eleven-gons whose vertices lie in an ideal of index 3 (generated by $1 \pm \omega$ ) in the Eisenstein integers determine non-negativelycurved triangulations of $S^{2}$. Each valley between star-tips is folded together to form the triangulation; the star-tips come together at the base vertex. If the inner vertices of the 11-pointed star are closer to the two adjacent star-tips than to any other star-tips, this is the canonical 11-gon for the triangulation based at the given vertex.

Actually, we will enlarge $S(P)$ to a surface $F(P)$ (resembling a flower) by adjoining sectors of circles of with apex at each vertex $v_{i}(i>0)$ of $S(P)$ and angle equal to the curvature at $v_{i}$ in $P$, so that the resulting surface is locally Euclidean everywhere in its interior (as in figure 14).

The minimum distance within $S(P)$ of any point in $S(P)$ from one star-points that assemble at $v_{0}$ is equal to the distance of its image in $P$ from $v_{0}$. Let $Q \subset S(P) \subset F(P)$ be the set of points whose minimum distance to $\partial F(P)$ is attained at 3 or more points on $\partial F(P)$. Then $Q$ includes $\left\{v_{1}, \ldots, v_{n}\right\}$, as well as the vertices for the Voronoi diagram of the star tips within $S(P)$. Let $R$ be the collection of open segments consisting of points whose minimum distance to $\partial F(P)$ is attained at two points of $\partial F(P)$; they are the edges of a tree, whose vertex set is $Q$. We decompose $S(P)$ into dart-shaped quadrilaterals, consisting of union of the two minimum-length arcs from points in an edge $\alpha \in R$ to $\partial F(P)$ (see figure 16). We'll call this dart $D(\alpha)$. Let $\theta(e)$ be the angle of $D(e)$ at either of its two wingtips (vertices that are not vertics of $e$ ). Note that

$$
\sum_{e \in R} \theta(e)=1 / 2\left(2 \pi-\kappa\left(v_{0}\right)\right)
$$




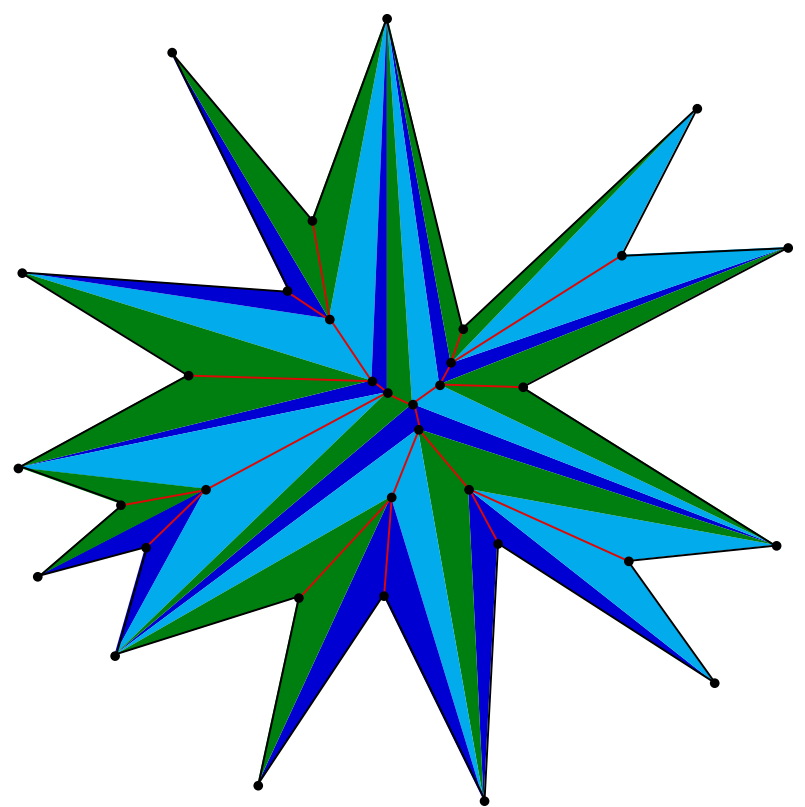

Figure 16: An irregular icosahedron sliced and flattened. A regular point $\star$ was chosen on a Euclidean cone-metric for $S^{2}$ having 12 cone points of curvature $\pi / 3$. The surface has been cut along shortest geodesics from $\star$ to each of the cone points, and flattened into the plane to form a 24 -gon resembling a star. The polygon has been subdivided into 45 dart-shaped quadrilaterals. Each quadrilateral is obtained from an edge of the cut locus of the original icosahedron $(=$ the Voronoi diagram for the $\star$-tips, after cutting) by suspending to its two closest $\star$-tips.

that is, half the cone angle at the base vertex.

For any vertex $q \in Q$, let $D(q) \subset F(P)$ be the maximal disk in $F(P)$ centered about $q_{i}$. If $q_{1}$ and $q_{2}$ are the endpoints of $e \in R$, then the angle between the bounding circles of $D\left(q_{1}\right)$ and $D\left(q_{2}\right)$ is $\theta(e)$.

Proposition 7.1 $F(P)$ has an isometric embedding in the plane $\mathbb{E}^{2}$.

Proof The developing map $f: F(P) \rightarrow \mathbb{E}^{2}$ into the plane is an isometric immersion. To show that it is an embedding, it will suffice to establish that $f$ restricted to the boundary $\partial F(P)$ is an embedding.

The boundary $\partial F(P)$ is composed of inward-curving circle arcs that meet at outward-bending angles. For each edge $e \in R$, there is a pair of these angles, where $\partial F(P)$ turns by an angle $\theta(e)$. For any two points $x, y \in \partial F(P)$, there is at least one path along $\partial F(P)$ where these bending angles sum to no more than $\pi$. 


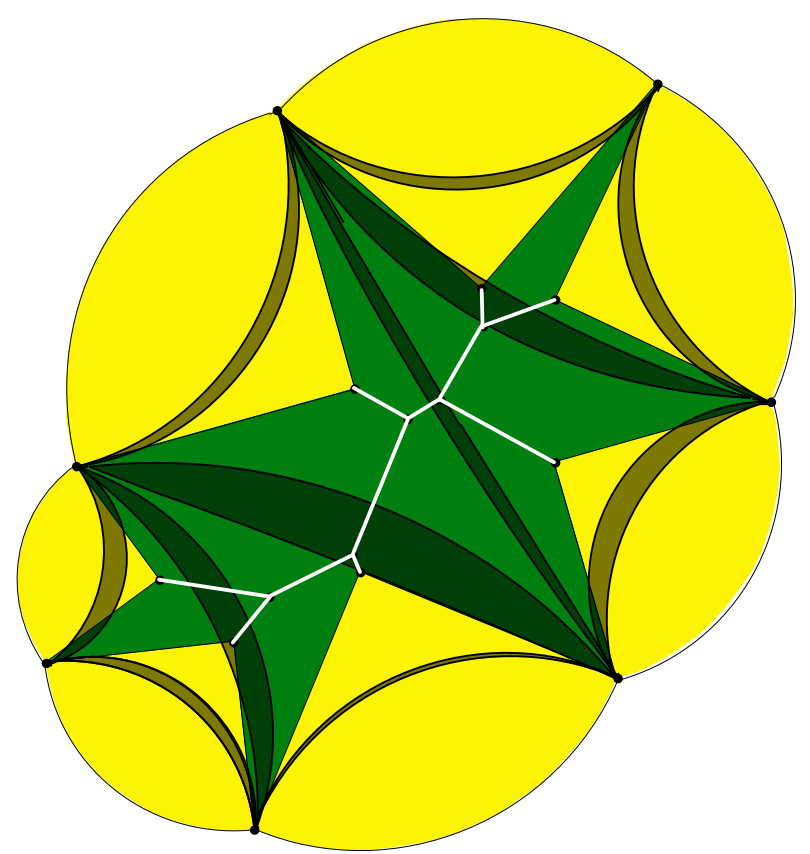

Figure 17: A hyperbolic view associated with the cut-open polyhedron. From the point of view of 3-dimensional hyperbolic geometry, if this figure is interpreted as lying on the boundary of upper half-space, the convex hull of its complement is the union of the hemispherical bubbles which rest on it. The boundary of the convex hull (with geometry induced from the upper half-space metric $\left.d s^{2}=1 / z^{2}\left(d x^{2}+d y^{2}+d z^{2}\right)\right)$ is isometric with the hyperbolic plane, bent into hyperbolic 3-space. The sum of all bending angles is one half the cone angle at the base point (assembled from the tips of stars). Any immersion of the hyperbolic plane which has total bending measure less than $\pi$ is an embedding, so this plane is embedded. There are immersed planes with total bending any number greater than $\pi$ which are not embedded.

For an immersion of a disk in $\mathbb{E}^{2}$ to fail to be an embedding, any innermost arc whose endpoints are identified by the immersion must have total curvature at least $-\pi$ when orientations are chosen so that the total curvature of the entire boundary is $2 \pi$. This is clearly impossible in our situation, so $f$ is actually an embedding.

Remark This proposition can be rephrased in terms of 3-dimensional hyperbolic geometry: any pleated immersion $f: \mathbb{H}^{2} \rightarrow \mathbb{H}^{3}$ with positive bending measure whose integral on any geodesic is no greater than $\pi$ is an embedding. This is related to the inequality of Sullivan analyzed and refined by Epstein and Marden in [4], and also to the global characterization of bending for convex polyhedra of Rivin and Hodgson [10], see Rivin [9] for a related inequality 
for convex hyperbolic polyhedra.

The $n$-dimensional associahedron is a polyhedron whose vertices are labelled by triangulations of an $(n+3)$-gon using only vertices of the polygon, and whose $k$-cells are labelled by subdivisions of the $(n+3)$-gon obtained by removing $k$ edges from a triangulation. They can be thought of as describing all ways to parenthesize or associate a product of $n+2$ symbols. The associahedron is a convex polyhedron in $n$-space that arises in a variety of mathematical context including the theory of loop spaces, Teichmüller theory and numerous combinatorial settings. The numbers of triangulations are called Catalan numbers.

If cone angles at the $n$ points of $P$ are fixed, the angles $\theta(e)$ that can occur for our dart quadrilaterals can be described by mapping the $n-1$ star tips of $S(P)$ to the vertices of a regular $(n-1)$-gon, mapping each dart quadrilateral $D(e)$ to an edge or a chord of this polygon, and labelling each edge by the angle $\theta(e)$. (In terms of hyperbolic geometry, this is an element of the measured lamination space for the ideal polygonal orbifold $(\infty, \infty, \ldots, \infty)$.) This determines a point in a polyhedron $\mathcal{F} n$ closely related to an associahedron, namely, the join of the boundary of the dual of the $(n-4)$-dimensional associahedron with the $(n-2)-$ simplex. (When the measure on the boundary of the polygon is zero, we get a point on the boundary of the dual of the $(n-4)$-dimensional associahedron. Measures on the polygon itself with fixed total weight form an $(n-2)$-simplex.)

The set of all possible functions $\theta(e)$ (which we refer to as measures, after the usage in hyperbolic geometry and Teichmüller theory) can be described globally as a convex polyhedron using dual train track coordinates, as follows: rotate a copy of the regular $(n-1)$-gon $1 /(2 n-2)$ th of a revolution so it is out of phase with itself. Choose any triangulation of this rotated polygon, using only its vertices. For each edge $f$ of this triangulation, let $m(f)$ be the sum of $\theta(e)$ where $e$ intersects $f$. For any triangle with sides $f, g, h$, the quantities $m(f)$, $m(g)$ and $m(h)$ satisfy the three triangle inequalities $m(f)+m(g) \geq m(h)$ etc. These measures are subject to one linear constraint, namely, the sum of $m(f)$ where $f$ ranges over the edges of the rotated polygon adds to the cone angle at the base vertex $v_{0}$.

For any set of numbers $\{m(f)\}$ satisfying the linear equation and linear inequalities, a measured lamination having total measure $\pi-\alpha_{0} / 2$, where $\alpha_{0}$ is the cone angle at $v_{0}$, can be reconstructed by a simple method familiar in the theory of measured foliations or normal curves on surfaces, by first solving for the picture in each triangle of the rotated polygon, then gluing the triangles together. From this measured lamination and from the specification of cone angles (in order) at $v_{1}, \ldots v_{n-1}$, a star polygon in the plane can be constructed 
recursively, using the principle that the shape of any dart quadrilateral $D(e)$ is determined from $\theta(e)$ together with either of its other two angles. This star polygon is determined up to similarity. When glued together it forms a cone manifold with specified cone-angles.

If all cone angles are equal, and if we are not distinguishing shapes that are the same up to permutation of the labels of cone points $v_{1}, \ldots v_{n-1}$, then we must divide $\mathcal{F}$ by action of the group of order $n-1$ rotations. The faces of $\mathcal{F}$ correspond to measures $\theta$ where one of the edges of the $(n-1)$-gon has measure 0 . Geometrically, this means that one of the cone points $v_{i}, i>0$ has two or more shortest paths on $P$ to $v_{0}$. We could cut $P$ open along either of these shortest paths. In $S(P)$, this means one of the "inside" vertices of the star has three or more shortest paths to the tip vertices: two are sides of $S(P)$, and at least one is interior to $S(P)$. You can cut $S(P)$ along such an edge, and rotate one resulting chunk with respect to the other, to obtain a new shape $S^{\prime}(P)$ with vertices in a permuted order.

To also insist on allowing change of base point requires a further much less direct equivalence relation. If the cone angles $\alpha_{1}, \ldots, \alpha_{n-1}$ are not all the same, then to get all possible cone-metrics, we need one copy of $\mathcal{F}$ for each ordering of the cone angles up to cyclic permutation.

\section{Teichmüller space interpretation}

Each element of $C\left(\alpha_{1}, \ldots, \alpha_{n}\right)$ determines a point in a certain finite sheeted covering of the modular orbifold for the $n$-punctured sphere. (The covering corresponds to the subgroup of the modular group for the $n$-punctured sphere which preserves the cone angles): the map consists of forgetting the metric, and remembering only the conformal structure.

By the uniformization theorem, each of these metrics is equivalent to a metric obtained by deleting $n$ points from the Riemann sphere $\hat{\mathbb{C}}$. The resulting configuration of $n$ points in $\hat{\mathbb{C}}$ is unique up to Möbius transformations.

Proposition 8.1 The map from $C\left(\alpha_{1}, \ldots, \alpha_{n}\right)$ is a homeomorphism.

Proof In fact, there is an explicit formula for the inverse map, going from a configuration of $n$ points on $\hat{\mathbb{C}}$ together with the curvatures at those points to a Euclidean cone-manifold with the given conformal structure. The formula is essentially the same as the Schwarz-Christoffel formula for uniformizing a 
rectilinear polygon. (See [12] for an analysis of these and other cone-manifold structures.)

The idea is to think of the construction of a Euclidean cone metric on $\hat{\mathbb{C}}$ in terms of its developing map $h$. Consider a collection $\left\{y_{i}\right\}$ of points in $\hat{\mathbb{C}}$, with desired curvatures $\left\{\alpha_{i}\right\}$. Let $P$ be the punctured Riemann sphere $\hat{\mathbb{C}}-\left\{y_{i}\right\}$. The developing map $h$ is not uniquely determined on $P$, and it is only defined on the universal cover $\tilde{P}$, but any two choices differ by a complex affine transformation. Therefore, the pre-Schwarzian of $h$, that is, $S=h^{\prime \prime} / h^{\prime}$, is uniquely determined by the metric, and it is defined on $P$, not just on the universal cover of $P$. The Euclidean metric can be easily reconstructed if we are given $S$, because once we choose an initial value and derivative for the developing map $h$ at one point on $\tilde{P}$, we can integrate the differential equation $h^{\prime \prime}=S h^{\prime}$ to determine it everywhere else.

How can we determine $S$ ? Consider a cone, with curvature $\alpha$ at the its apex. If a cone is conformally mapped to $\mathbb{C}$ with its apex going to the origin, the developing map is $z \mapsto z^{1-\frac{\alpha}{2 \pi}}$. The pre-Schwarzian for this map is $-\frac{\alpha}{2 \pi} z^{-1}$. It follows that the pre-Schwarzian for the developing map of any Euclidean conemetric with a cone point having curvature $\alpha$ will have a pole at the cone point, with residue $-\frac{\alpha}{2 \pi} z^{-1}$. Conversely, if the pre-Schwarzian of some function $h$ has a pole of this type at any point in $\hat{\mathbb{C}}$, then $h$ will locally be the developing map for a Euclidean structure with a cone point of angle $\alpha$. (To see this, observe that the analytic continuation of $h$ around the pole differs by postcomposition with an affine transformation. Using this information, one can make a local conformal change of coordinates in the domain so that $h$ has the form $z \mapsto z^{1-\frac{\alpha}{2 \pi}}$, where $\alpha$ is not necessarily real. From this, one sees that the pre-Schwarzian has a pole at the singularity with residue $\alpha / 2 \pi$.)

We may as well assume that the the $\left\{y_{i}\right\}$ are in the finite part of $\hat{\mathbb{C}}$. Define

$$
S=\sum_{i}-\frac{\alpha}{2 \pi}\left(z-y^{-1}\right) \text {. }
$$

Computation shows that in a coordinate patch $w=z^{-1}$ for a neighborhood of $\infty$, the pre-Schwarzian in terms of the variable $w$ is holomorphic if and only if $S$ behaves asymptotically like $-2 z^{-1}$. This is satisfied in our case, since the sum of the $\alpha_{i}$ is $4 \pi$. The condition that $S$ is holomorphic on $P$, and that it has the given behaviour at the cone points and at $\infty$, uniquely determines $S$.

$S$ determines a complex affine structure on $P$. Since the fundamental group of $P$ is generated by loops going around the punctures, and since the holonomy around these loops is isometric, the affine structure is compatible with a Euclidean structure, well-defined up to scaling. 
Thus, we may think of $C\left(\alpha_{1}, \ldots, \alpha_{n}\right)$ as a certain geometric interpretation of modular space. The completions $\bar{C}\left(\alpha_{1}, \ldots, \alpha_{n}\right)$ have a topology which depends on the comparisons of sums of subsets of the $\alpha_{i}$ with $2 \pi$. It is almost never agrees with the standard compactification of the modular space. However, there are only a finite number of possible possibilities for the topology — it is curious that we thus obtain several parameter families of complex hyperbolic structures on the Teichmüller space, and several parameter families of complex hyperbolic cone-manifolds on the various $\bar{C}\left(\alpha_{1}, \ldots, \alpha_{n}\right)$, with varying cone angles.

Is there any similar phenomenon for the Teichmüller spaces of other surfaces, particularly closed surfaces? The surface of genus 2 has the same modular space as the six-punctured sphere, so for that particular case, the construction carries over. I don't know how to extend it to surfaces of higher genus.

\section{References}

[1] D Cooper, W P Thurston, Triangulating 3-manifolds using 5 vertex links, Topology, 27 (1988) 23-25

[2] P Deligne, G D Mostow, Monodromy of hypergeometric functions and nonlattice integral monodromy, Inst. Hautes Études Sci. Publ. Math. 63 (1986) 5-89

[3] P Deligne, GD Mostow, Commensurabilities among lattices in $\mathrm{PU}(1, n)$, Annals of Mathematics Studies, 132, Princeton University Press, Princeton, NJ (1993)

[4] D B A Epstein, A Marden, Convex hulls in hyperbolic space, a theorem of Sullivan, and measured pleated surfaces, from: "Analytical and geometric aspects of hyperbolic space (Coventry/Durham, 1984)", Cambridge Univ. Press, Cambridge (1987) 113-253

[5] G D Mostow, Generalized Picard lattices arising from half-integral conditions Inst. Hautes Études Sci. Publ. Math. 63 (1986) 91-106

[6] E Picard, Sur les fonctions hyperfuchsiennes provenant des séries hypergéometriques de deux variables, Ann. ENS III, 2 (1885) 357-384

[7] E Picard, Sur une extension aux fonctions de deux variables du problème de Riemann relatif aux fonctions hypergéometriques, Bull. Soc. Math. Fr. 15 (1887) $148-152$

[8] Igor Rivin, Euclidean structures on simplicial surfaces and hyperbolic volume, Annals of Math. 139 (1994) 553-580

[9] Igor Rivin, A characterization of ideal polyhedra in hyperbolic 3-space, Annals of Math. 143 (1996) 51-70

[10] Igor Rivin, Craig D Hodgson, A characterization of compact convex polyhedra in hyperbolic 3 -space, Invent. Math. 111 (1993) 77-111 
[11] William P Thurston, Geometry and Topology of Three-Manifolds, Princeton lecture notes (1979) http://www.msri.org/publications/books/gt3m

[12] Marc Troyanov, Prescribing curvature on compact surfaces with conical singularities, Trans. Amer. Math. Soc. 324 (1991) 793-821

Mathematics Department

University of California at Davis

Davis, CA 95616, USA

Email: wpt@math.ucdavis.edu

Received: 15 November 1997 Revised: 27 November 1998

\section{Appendix: 94 orbifolds}

We give below a list of the examples of the spaces $C\left(\alpha_{1}, \ldots, \alpha_{n}\right)$ which are orbifolds, for $n \geq 5$. When $n=3$ there is only one example for each feasible triple of cone angles, and for $n=4$ there are infinitely many examples. In fact, every triangle group in the hyperbolic plane can be interpreted as the modular space for families of tetrahedra. In general, the $\alpha_{i}$ are of the form $\frac{2 \pi p}{q}$, for $p$ and $q$ integers. For each example, we list the least denominator $q$ and the sequence of numerators $p_{i}$. We also list the degree of the number field containing the roots of unity $\exp \left(\frac{2 \pi p_{i}}{q}\right)$ (that is, the number of integers less than $q$ relatively prime to $q$ ). We list also three additional bits of information:

(arithmetic) Is the orbifold arithmetic (AR) or non-arithmetic (NR)?

(pure) Is the completion of the covering of the modular space whose fundamental group is the pure braid group an orbifold $(\mathrm{P})$, or are some interchanges of pairs of cone points needed to make the orbifold (I)?

(compact) Is the orbifold compact (C) or non-compact (N)?

The question of arithmeticity hinges on the signatures of the Hermitian forms obtained when we conjugate the curvatures at the cone points (considered as roots of unity) by the Galois automorphisms. If all the other signatures are negative definite or positive definite, the group is arithmetic; otherwise not. The other two questions are more obvious.

These examples were enumerated by a routine computer program, which checks all possibilities having a given least common denominator $q$. The enumeration was not rigorously verified (even though it should not be hard to do so and search more 'intelligently' at the same time) but was a simple check of all denominators through 999 in a few minutes of computer time. Mostow has rigorously enumerated examples by hand, so this table can be regarded as just a check. 


\begin{tabular}{|c|c|c|c|c|c|c|c|c|c|c|c|c|c|c|c|c|}
\hline & Denominator & & & & $\mathrm{me}$ & tor: & & & & & & & degree & arithmetic? & pure? & $\begin{array}{l}\text { compact? } \\
\end{array}$ \\
\hline 1. & 3 & 1 & 1 & 1 & 1 & 1 & 1 & & & & & & 2 & $\mathrm{AR}$ & $\mathrm{P}$ & $\mathrm{N}$ \\
\hline 2. & 3 & 2 & 1 & 1 & 1 & 1 & & & & & & & 2 & AR & $\mathrm{P}$ & $\mathrm{N}$ \\
\hline 3. & 4 & 1 & 1 & 1 & 1 & 1 & 1 & 1 & 1 & & & & 2 & $\mathrm{AR}$ & $\mathrm{P}$ & $\mathrm{N}$ \\
\hline 4. & 4 & 2 & 1 & 1 & 1 & 1 & 1 & 1 & & & & & 2 & $\mathrm{AR}$ & $\mathrm{P}$ & $\mathrm{N}$ \\
\hline 5. & 4 & 3 & 1 & 1 & 1 & 1 & 1 & & & & & & 2 & $\mathrm{AR}$ & $\mathrm{P}$ & $\mathrm{N}$ \\
\hline 6. & 4 & 2 & 2 & 1 & 1 & 1 & 1 & & & & & & 2 & $\mathrm{AR}$ & $\mathrm{P}$ & $\mathrm{N}$ \\
\hline 7. & 4 & 3 & 2 & 1 & 1 & 1 & & & & & & & 2 & $\mathrm{AR}$ & $\mathrm{P}$ & $\mathrm{N}$ \\
\hline 8. & 4 & 2 & 2 & 2 & 1 & 1 & & & & & & & 2 & $\mathrm{AR}$ & $\mathrm{P}$ & $\mathrm{N}$ \\
\hline 9. & 5 & 2 & 2 & 2 & 2 & 2 & & & & & & & 4 & $\mathrm{AR}$ & $\mathrm{P}$ & $\mathrm{C}$ \\
\hline 10. & 6 & 1 & 1 & 1 & 1 & 1 & 1 & 1 & 1 & 1 & 1 & $\begin{array}{ll}1 & 1\end{array}$ & 2 & $\mathrm{AR}$ & I & $\mathrm{N}$ \\
\hline 11. & 6 & 2 & 1 & 1 & 1 & 1 & 1 & 1 & 1 & 1 & 1 & 1 & 2 & $\mathrm{AR}$ & I & $\mathrm{N}$ \\
\hline 12. & 6 & 3 & 1 & 1 & 1 & 1 & 1 & 1 & 1 & 1 & 1 & & 2 & $\mathrm{AR}$ & I & $\mathrm{N}$ \\
\hline 13. & 6 & 2 & 2 & 1 & 1 & 1 & 1 & 1 & 1 & 1 & 1 & & 2 & $\mathrm{AR}$ & I & $\mathrm{N}$ \\
\hline 14. & 6 & 4 & 1 & 1 & 1 & 1 & 1 & 1 & 1 & 1 & & & 2 & $\mathrm{AR}$ & I & $\mathrm{N}$ \\
\hline 15. & 6 & 3 & 2 & 1 & 1 & 1 & 1 & 1 & 1 & 1 & & & 2 & $\mathrm{AR}$ & I & $\mathrm{N}$ \\
\hline 16. & 6 & 5 & 1 & 1 & 1 & 1 & 1 & 1 & 1 & & & & 2 & $\mathrm{AR}$ & I & $\mathrm{N}$ \\
\hline 17. & 6 & 2 & 2 & 2 & 1 & 1 & 1 & 1 & 1 & 1 & & & 2 & $\mathrm{AR}$ & I & $\mathrm{N}$ \\
\hline 18. & 6 & 4 & 2 & 1 & 1 & 1 & 1 & 1 & 1 & & & & 2 & $\mathrm{AR}$ & I & $\mathrm{N}$ \\
\hline 19. & 6 & 3 & 3 & 1 & 1 & 1 & 1 & 1 & 1 & & & & 2 & $\mathrm{AR}$ & I & $\mathrm{N}$ \\
\hline 20 . & 6 & 3 & 2 & 2 & 1 & 1 & 1 & 1 & 1 & & & & 2 & $\mathrm{AR}$ & I & $\mathrm{N}$ \\
\hline 21. & 6 & 5 & 2 & 1 & 1 & 1 & 1 & 1 & & & & & 2 & $\mathrm{AR}$ & I & $\mathrm{N}$ \\
\hline 22 & 6 & 4 & 3 & 1 & 1 & 1 & 1 & 1 & & & & & 2 & $\mathrm{AR}$ & I & $\mathrm{N}$ \\
\hline 23. & 6 & 2 & 2 & 2 & 2 & 1 & 1 & 1 & 1 & & & & 2 & $\mathrm{AR}$ & I & $\mathrm{N}$ \\
\hline 24 & 6 & 4 & 2 & 2 & 1 & 1 & 1 & 1 & & & & & 2 & $\mathrm{AR}$ & I & $\mathrm{N}$ \\
\hline 25. & 6 & 3 & 3 & 2 & 1 & 1 & 1 & 1 & & & & & 2 & $\mathrm{AR}$ & I & $\mathrm{N}$ \\
\hline 26. & 6 & 5 & 3 & 1 & 1 & 1 & 1 & & & & & & 2 & $\mathrm{AR}$ & I & $\mathrm{N}$ \\
\hline 27. & 6 & 4 & 4 & 1 & 1 & 1 & 1 & & & & & & 2 & $\mathrm{AR}$ & I & $\mathrm{N}$ \\
\hline 28. & 6 & 3 & 2 & 2 & 2 & 1 & 1 & 1 & & & & & 2 & $\mathrm{AR}$ & I & $\mathrm{N}$ \\
\hline 29. & 6 & 5 & 2 & 2 & 1 & 1 & 1 & & & & & & 2 & AR & I & $\mathrm{N}$ \\
\hline 30. & 6 & 4 & 3 & 2 & 1 & 1 & 1 & & & & & & 2 & $\mathrm{AR}$ & I & $\mathrm{N}$ \\
\hline 31. & 6 & 3 & 3 & 3 & 1 & 1 & 1 & & & & & & 2 & $\mathrm{AR}$ & I & $\mathrm{N}$ \\
\hline 32. & 6 & 5 & 4 & 1 & 1 & 1 & & & & & & & 2 & $\mathrm{AR}$ & I & $\mathrm{N}$ \\
\hline 33. & 6 & 2 & 2 & 2 & 2 & 2 & 1 & 1 & & & & & 2 & $\mathrm{AR}$ & I & $\mathrm{N}$ \\
\hline 34. & 6 & 4 & 2 & 2 & 2 & 1 & 1 & & & & & & 2 & $\mathrm{AR}$ & I & $\mathrm{N}$ \\
\hline 35. & 6 & 3 & 3 & 2 & 2 & 1 & 1 & & & & & & 2 & $\mathrm{AR}$ & I & $\mathrm{N}$ \\
\hline 36. & 6 & 5 & 3 & 2 & 1 & 1 & & & & & & & 2 & $\mathrm{AR}$ & I & $\mathrm{N}$ \\
\hline 37. & 6 & 4 & 4 & 2 & 1 & 1 & & & & & & & 2 & AR & I & $\mathrm{N}$ \\
\hline 38. & 6 & 4 & 3 & 3 & 1 & 1 & & & & & & & 2 & $\mathrm{AR}$ & I & $\mathrm{N}$ \\
\hline 39. & 6 & 3 & 2 & 2 & 2 & 2 & 1 & & & & & & 2 & $\mathrm{AR}$ & $\mathrm{P}$ & $\mathrm{N}$ \\
\hline 40. & 6 & 5 & 2 & 2 & 2 & 1 & & & & & & & 2 & $\mathrm{AR}$ & $\mathrm{P}$ & $\mathrm{N}$ \\
\hline 41. & 6 & 4 & 3 & 2 & 2 & 1 & & & & & & & 2 & $\mathrm{AR}$ & $\mathrm{P}$ & $\mathrm{N}$ \\
\hline 42 & 6 & 3 & 3 & 3 & 2 & 1 & & & & & & & 2 & $\mathrm{AR}$ & $\mathrm{P}$ & $\mathrm{N}$ \\
\hline 43. & 6 & 3 & 3 & 2 & 2 & 2 & & & & & & & 2 & $\mathrm{AR}$ & $\mathrm{P}$ & $\mathrm{N}$ \\
\hline 44. & 8 & 3 & 3 & 3 & 3 & 3 & 1 & & & & & & 4 & $\mathrm{AR}$ & $\mathrm{P}$ & $\mathrm{C}$ \\
\hline 45 & 8 & 6 & 3 & 3 & 3 & 1 & & & & & & & 4 & $\mathrm{AR}$ & $\mathrm{P}$ & $\mathrm{C}$ \\
\hline 46. & 8 & 5 & 5 & 2 & 2 & 2 & & & & & & & 4 & $\mathrm{AR}$ & $\mathrm{P}$ & $\mathrm{C}$ \\
\hline 47. & 8 & 4 & 3 & 3 & 3 & 3 & & & & & & & 4 & $\mathrm{AR}$ & $\mathrm{P}$ & $\mathrm{C}$ \\
\hline
\end{tabular}




\begin{tabular}{|c|c|c|c|c|c|c|c|c|c|c|c|c|}
\hline & Denominator & & & & mera & & & & degree & arithmetic? & pure? & compact? \\
\hline 48. & 9 & 4 & 4 & 4 & 4 & 2 & & & 6 & $\mathrm{AR}$ & $\mathrm{P}$ & $\mathrm{C}$ \\
\hline 49. & 10 & 7 & 4 & 4 & 4 & 1 & & & 4 & AR & $\mathrm{P}$ & C \\
\hline 50 & 10 & 3 & 3 & 3 & 3 & 3 & 3 & 2 & 4 & AR & I & $\mathrm{C}$ \\
\hline 51. & 10 & 6 & 3 & 3 & 3 & 3 & 2 & & 4 & $\mathrm{AR}$ & I & C \\
\hline 52 & 10 & 9 & 3 & 3 & 3 & 2 & & & 4 & $\mathrm{AR}$ & I & $\mathrm{C}$ \\
\hline 53. & 10 & 6 & 6 & 3 & 3 & 2 & & & 4 & $\mathrm{AR}$ & I & $\mathrm{C}$ \\
\hline 54. & 10 & 5 & 3 & 3 & 3 & 3 & 3 & & 4 & AR & I & $\mathrm{C}$ \\
\hline 55. & 10 & 8 & 3 & 3 & 3 & 3 & & & 4 & $\mathrm{AR}$ & I & $\mathrm{C}$ \\
\hline 56. & 10 & 6 & 5 & 3 & 3 & 3 & & & 4 & $\mathrm{AR}$ & I & $\mathrm{C}$ \\
\hline 57. & 12 & 8 & 5 & 5 & 5 & 1 & & & 4 & $\mathrm{AR}$ & $\mathrm{P}$ & $\mathrm{C}$ \\
\hline 58. & 12 & 7 & 7 & 2 & 2 & 2 & 2 & 2 & 4 & $\mathrm{AR}$ & I & $\mathrm{C}$ \\
\hline 59. & 12 & 9 & 7 & 2 & 2 & 2 & 2 & & 4 & $\mathrm{AR}$ & I & $\mathrm{C}$ \\
\hline 60 & 12 & 7 & 7 & 4 & 2 & 2 & 2 & & 4 & $\mathrm{AR}$ & I & $\mathrm{C}$ \\
\hline 61. & 12 & 11 & 7 & 2 & 2 & 2 & & & 4 & $\mathrm{AR}$ & I & $\mathrm{C}$ \\
\hline 62 & 12 & 9 & 9 & 2 & 2 & 2 & & & 4 & $\mathrm{AR}$ & I & $\mathrm{C}$ \\
\hline 63. & 12 & 9 & 7 & 4 & 2 & 2 & & & 4 & AR & I & $\mathrm{C}$ \\
\hline 64 & 12 & 7 & 7 & 6 & 2 & 2 & & & 4 & $\mathrm{AR}$ & I & $\mathrm{C}$ \\
\hline 65. & 12 & 7 & 7 & 4 & 4 & 2 & & & 4 & $\mathrm{AR}$ & $\mathrm{P}$ & C \\
\hline 66. & 12 & 7 & 5 & 3 & 3 & 3 & 3 & & 4 & NA & $\mathrm{P}$ & $\mathrm{N}$ \\
\hline 67. & 12 & 5 & 5 & 5 & 3 & 3 & 3 & & 4 & $\mathrm{AR}$ & $\mathrm{P}$ & $\mathrm{C}$ \\
\hline 68. & 12 & 10 & 5 & 3 & 3 & 3 & & & 4 & $\mathrm{AR}$ & $\mathrm{P}$ & $\mathrm{C}$ \\
\hline 69. & 12 & 8 & 7 & 3 & 3 & 3 & & & 4 & NA & $\mathrm{P}$ & $\mathrm{C}$ \\
\hline 70 & 12 & 8 & 5 & 5 & 3 & 3 & & & 4 & $\mathrm{AR}$ & $\mathrm{P}$ & $\mathrm{C}$ \\
\hline 71. & 12 & 7 & 6 & 5 & 3 & 3 & & & 4 & NA & $\mathrm{P}$ & $\mathrm{N}$ \\
\hline 72 & 12 & 6 & 5 & 5 & 5 & 3 & & & 4 & $\mathrm{AR}$ & $\mathrm{P}$ & $\mathrm{C}$ \\
\hline 73. & 12 & 7 & 5 & 4 & 4 & 4 & & & 4 & NA & $\mathrm{P}$ & $\mathrm{N}$ \\
\hline 74 & 12 & 6 & 5 & 5 & 4 & 4 & & & 4 & NA & $\mathrm{P}$ & $\mathrm{C}$ \\
\hline 75 & 12 & 5 & 5 & 5 & 5 & 4 & & & 4 & $\mathrm{AR}$ & $\mathrm{P}$ & $\mathrm{C}$ \\
\hline 76. & 14 & 11 & 5 & 5 & 5 & 2 & & & 6 & $\mathrm{AR}$ & I & $\mathrm{C}$ \\
\hline 77. & 14 & 8 & 5 & 5 & 5 & 5 & & & 6 & $\mathrm{AR}$ & I & $\mathrm{C}$ \\
\hline 78. & 15 & 8 & 6 & 6 & 6 & 4 & & & 8 & NA & $\mathrm{P}$ & $\mathrm{C}$ \\
\hline 79. & 18 & 11 & 8 & 8 & 8 & 1 & & & 6 & $\mathrm{AR}$ & $\mathrm{P}$ & $\mathrm{C}$ \\
\hline 80 & 18 & 13 & 7 & 7 & 7 & 2 & & & 6 & NA & I & $\mathrm{C}$ \\
\hline 81. & 18 & 10 & 10 & 7 & 7 & 2 & & & 6 & $\mathrm{AR}$ & I & C \\
\hline 82 & 18 & 14 & 13 & 3 & 3 & 3 & & & 6 & $\mathrm{AR}$ & I & $\mathrm{C}$ \\
\hline 83. & 18 & 10 & 7 & 7 & 7 & 5 & & & 6 & $\mathrm{AR}$ & I & $\mathrm{C}$ \\
\hline 84 & 18 & 8 & 7 & 7 & 7 & 7 & & & 6 & NA & I & $\mathrm{C}$ \\
\hline 85 & 20 & 14 & 11 & 5 & 5 & 5 & & & 8 & NA & $\mathrm{P}$ & $\mathrm{C}$ \\
\hline 86. & 20 & 13 & 9 & 6 & 6 & 6 & & & 8 & NA & I & $\mathrm{C}$ \\
\hline 87. & 20 & 10 & 9 & 9 & 6 & 6 & & & 8 & NA & I & $\mathrm{C}$ \\
\hline 88. & 24 & 19 & 17 & 4 & 4 & 4 & & & 8 & NA & I & $\mathrm{C}$ \\
\hline 89. & 24 & 14 & 9 & 9 & 9 & 7 & & & 8 & NA & $\mathrm{P}$ & $\mathrm{C}$ \\
\hline 90. & 30 & 26 & 19 & 5 & 5 & 5 & & & 8 & $\mathrm{AR}$ & I & C \\
\hline 91. & 30 & 23 & 22 & 5 & 5 & 5 & & & 8 & NA & I & $\mathrm{C}$ \\
\hline 92. & 30 & 22 & 11 & 9 & 9 & 9 & & & 8 & $\mathrm{AR}$ & I & $\mathrm{C}$ \\
\hline 93. & 42 & 34 & 29 & 7 & 7 & 7 & & & 12 & NA & I & $\mathrm{C}$ \\
\hline 94. & 42 & 26 & 15 & 15 & 15 & 13 & & & 12 & NA & I & $\mathrm{C}$ \\
\hline
\end{tabular}




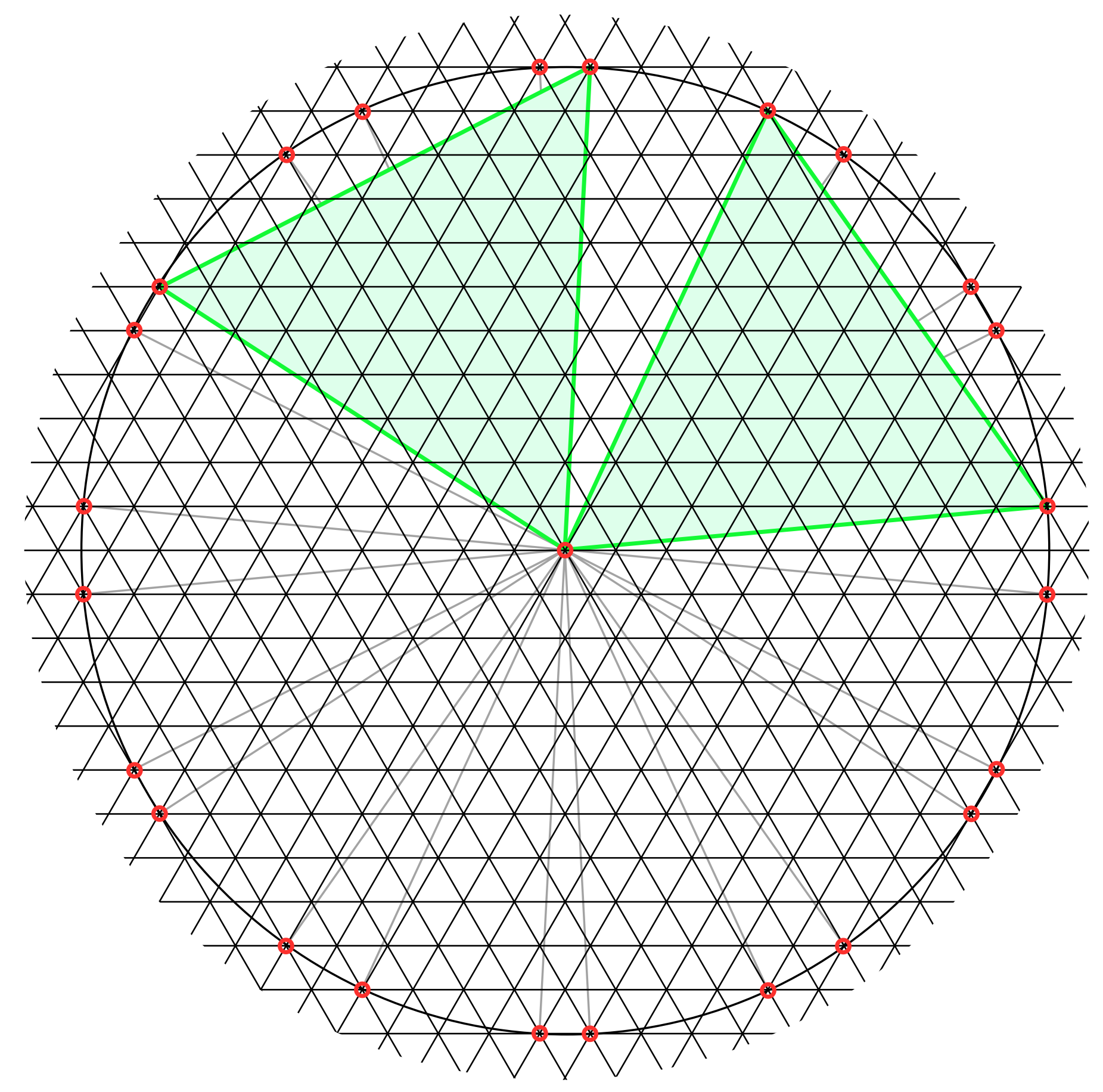

